SOGANG-HEP 197/95

\title{
On the Foundation of the Relativistic Dynamics with the Tachyon
}

\author{
Mu-In Park and Young-Jai Park \\ Department of Physics* and Basic Science Research Institute \\ Sogang University, C.P.O.Box 1142, Seoul 100-611, Korea
}

\begin{abstract}
The theoretical foundation of the object moving faster than light in vacuum (tachyon) is still missing or incomplete. Here we present the classical foundation of the relativistic dynamics including the tachyon. An anomalous sign-factor extracted from the transformation of $\sqrt{1-u^{2} / c^{2}}$ under the Lorentz transformation, which has been always missed in the usual formulation of the tachyon, has a crucial role in the dynamics of the tachyon. Due to this factor the mass of the tachyon transforms in the unusual way although the energy and momentum, which are defined as the conserved quantities in all uniformly moving systems, transform in the usual way as in the case of the object moving slower than light (bradyon). We show that this result can be also obtained from the least action approach. On the other hand, we show that the ambiguities for the description of the dynamics for the object moving with the velocity of light (luxon) can be consistently removed only by introducing a new dynamical variable. Furthermore, by using the fundamental definition of the momentum and energy we show that the zero-point energy for any kind of the objects, i.e., the tachyon, bradyon, and luxon, which has been known as the undetermined constant, should satisfy some constraints for consistency, and we note that this is essentially another novel relativistic effect. Finally, we remark about the several unsolved problems.
\end{abstract}

May 1995

* Electronic address: mipark, yjpark@physics.sogang.ac.kr 


\section{INTRODUCTION}

According to the Einstein's special theory of relativity, the object moving faster than light in vacuum [1] (tachyon after G. Feinberg [2]) can violate the causality, i.e., the relationship between the causes and effects has no absolute meaning for the observers moving uniformly with each others [3,4]. Moreover, when the formulas of the energy and momentum for the object moving slower than light (bradyon [5]) are directly applied to the tachyon, the energy and momentum of the tachyon become to be imaginary-valued $[4,6]$ implying that the tachyon is believed to be never detected by our ordinary bradyonic detectors if energy-momentum are also conserved in this process [7]. These were main objections to the existence of the tachyon. However, many years later, Bilaniuk-Deshpande-Sudarshan and Feinberg (BDSF) had suggested that these two difficulties can be avoided [2]: the first difficulty is avoided by introducing the imaginary-valued rest mass of the tachyon, which can not be ruled out in principle because the rest mass is not directly measurable quantity unless the object can be brought to rest, and by noting that there are no rest system for the tachyon according to the result of the usual Lorentz transformation. The second difficulty is avoided by introducing the so-called reinterpretation principle of " the negative energy state of the tachyon with reversed or backward time sequences " to be the positive energy state of the tachyon with ordinary or forward time sequences. However, the theoretical foundation of the suggestions of BDSF is still missing or incomplete although their suggestions were discussed by various authors [8], and further development on the quantum theory of the tachyon field theory [9] were followed. This is essentially due to the fact that their suggestions rely heavily on the formulas of the bradyon. In fact, the energy and momentum formulas for the bradyon can not be directly applied to the tachyon because some of the logics in the derivations of the formulas can not be true for the tachyon. Hence the separate derivations of the formulas for the tachyon from the first principles are required. Actually the incompleteness of the usual formulations was pointed out by several authors [10], but the complete formulation is still absent.

In this paper we present the classical foundation of the relativistic dynamics including the tachyon. In Sec. II the principles of the relativistic mechanics are reconsidered for the case that there are tachyons as well as the bradyons. An anomalous sign-factor in the transformation of $\sqrt{1-u^{2} / c^{2}}$ under the Lorentz transformation, which is unimportant for the bradyon and has been always missed in the usual formulation of the tachyon, has now a crucial role in the dynamics of the tachyon. Due to this novel sign-factor it is found from the consideration of the collision process that the proper-mass of the tachyon is not an absolute quantity in contrast to that of the bradyon but transforms as $\kappa^{\prime}=\operatorname{sign}\left\{1-\mathbf{u} \cdot \mathbf{v} / c^{2}\right\} \kappa$. Furthermore, we show that the tachyon's mass has a definite sign for a given speed in a uniformly moving system, but has

the sign changed depending on the tachyon's velocity and the relative motion of the systems $S$ 
and $S^{\prime}$.

On the other hand, by defining the momentum and energy as the conserved quantities of the presumed forms in all uniformly moving systems, we show that the zero-point energy $\epsilon_{a}$ for any kind of the objects of the both tachyon and bradyon, which has been known as the undetermined constant, should satisfy some constraints for consistency. Furthermore, especially for the case of $\epsilon_{a}=0$ for all $a$ as in the usual conventions, it is found that the energy and momentum, for the tachyon as well as the bradyon, satisfy the usual four-vector transformation such that the energy for the tachyon does not have the invariant sign of the energy, but the sign can be changed depending on the object's velocity and the relative motion of the two systems of coordinates $S$ and $S^{\prime}$ in contrast to that of the bradyon. However, we note that this transformation formulas can not be obtained from the usual formulation of the tachyon in contrast to the usual belief such that the original motivation of the BDSF's reinterpretation principle, if it applies to our Nature, can not be found in the usual formulation but, only in our new formulation of the tachyon.

In Sec. III we develop the least action approach to the tachyon dynamics by comparing the approach for the bradyon. Although it is not clear whether this approach can be also applied to the tachyon since the situation of $\Delta t=0$ for the time interval along the world-line can not be avoided for any tachyon such that there are some ambiguities in deriving the equation of motion. However, we show that this can be really also applied to the tachyon with the help of the reparametrization invariance of the action, and the essential results for the tachyon of the elementary approach of Sec. II can be also rederived in this approach.

In Sec. IV we develop the least action approach for the object moving with the velocity of light (luxon) [11]. We emphasize that a new dynamical variable should be introduced as well as the velocity variable in order to describe the luxon dynamics without any ambiguity. Their physical meaning is uncovered by comparing the Einstein relation for the photon. It is shown that in this approach with the help of these new variables, the usual energy-momentum relation for the luxon is proved without any ambiguity, and the energy and momentum of the luxon also satisfy the usual transformation like as the tachyon and bradyon, which has not been proved in the usual classical mechanical formulation. Moreover, with the help of the energy and momentum transformation under the Lorentz transformation, the zero-point energy for the luxon should satisfy the same constraints as in the case of the tachyon and bradyon considered in Sec. II, if we maintain the definition of the energy and momentum as the conserved quantities for all the systems of coordinates and all the possible process of the collision like as the case of the tachyon and bradyon. Especially, for the case of the photon it's zero-point energy should be zero due to the fact that it is clearly the strictly neutral particles.

Sec. $\mathrm{V}$ is devoted to summary and concluding remarks especially on the problems of causal- 
ity violation, the Čerenkov effect in the vacuum, and the quantization.

\section{THE PRINCIPLES OF RELATIVISTIC MECHANICS INCLUDING THE TACHYON}

The space and time of a system of coordinates, in which the equations of Newtonian mechanics hold well, and which will be denoted by stationary system $S$ hereafter, are essentially defined by the measuring rods and clocks at rest relatively to this system without references to the motions and properties of the measured object. Moreover, the transformation law of the space and time from a system $S$ to another system $S^{\prime}$ in uniform motion of translation relatively to the former with the relative speed to be smaller than that of light, is uniquely determined by postulating a) the two basic hypothesis, i.e., the principle of relativity and constancy of the velocity of light with b) some plausible assumptions on the structure of the space and time, i.e., the homogeneity of space and time, the isotropy of space and the validity of Euclidean geometry on this space and time, and c) tacit assumption that there exists at least one stationary system relatively to the observers for any motion of them.

In the usual formulation of the relativistic mechanics, if the momentum of the point objects

$$
\mathbf{p}=m(\mathbf{u}) \mathbf{u}
$$

is essentially defined to be the conserved quantity in total for any stationary system $S$, the mass function $m(\mathbf{u})$ is found to be

$$
m(\mathbf{u})=\frac{m_{0}}{\sqrt{1-u^{2} / c^{2}}} .
$$

Moreover, the quantity of the energy having the form

$$
E=m(\mathbf{u}) c^{2}
$$

is proved to be totally conserved [12], and as a result the principle of energy conservation is not independent on the principle of momentum conservation [13]. However, some of the usual methods of derivations of these formulas are valid only for the bradyon case essentially due to the non-existence of the rest system for the tachyon [13]. Therefore, we must carefully select the methods of derivation in order to include, from the start, the tachyon as well in the process of interaction of objects. Fortunately, there exists already an appropriate formulation for this purpose although the power of this formulation has been never well noticed [14]. Rather, this latter formulation has been believed to be worse than the former in the aspect of the economics 
of the logic because the latter formulation assumes one more condition, which is actually equivalent to the principle of energy conservation, on the mass function $m(\mathbf{u})$ in order to derive all the same results of the former formulation [15]. But, now, by using the latter formulation, we can discuss the mechanics of the tachyon from the start without any problem at the cost of the economy of the logic.

\section{A. The definition of the mass and the results drawn from it}

The theory of the mechanics can only be constructed by defining the mass of inertia of the point objects. In order to define the mass, let $S$ and $S^{\prime}$ be two systems of coordinates with the relative velocity $\mathbf{v}$, and consider a system of point objects composed of the tachyon as well as the bradyon in both $S$ and $S^{\prime}$. We fundamentally define the mass of inertia $m$ of each object in the system $S$ such that both

$$
\sum_{a} m_{a}
$$

and

$$
\sum_{a} m_{a} \mathbf{u}_{a}
$$

are conserved through all the possible processes of collision and in all the inertial systems of coordinates. Then, by representing $\mathbf{u}_{a}^{\prime}$ as the velocity of $a$-th object in $S^{\prime}$, the mass of inertia of each object in $S^{\prime}$ is defined such that both

$$
\sum_{a} m_{a}^{\prime}
$$

and

$$
\sum_{a} m_{a}^{\prime} \mathbf{u}_{a}^{\prime}
$$

are also conserved through all the possible processes of collision.

In order to find the explicit formula of the mass we first note that

$$
\begin{aligned}
\sqrt{1-u_{a}^{\prime 2} / c^{2}} & =\frac{\sqrt{1-u_{a}^{2} / c^{2}} \sqrt{1-v^{2} / c^{2}}}{\left|1-\mathbf{u}_{a} \cdot \mathbf{v} / c^{2}\right|} \\
& =\frac{\sqrt{1-u_{a}^{2} / c^{2}} \sqrt{1-v^{2} / c^{2}}}{\left\{1-\mathbf{u}_{a} \cdot \mathbf{v} / c^{2}\right\} \operatorname{sign}\left\{1-\mathbf{u}_{a} \cdot \mathbf{v} / c^{2}\right\}}
\end{aligned}
$$


from the well known usual velocity transformation formula

$$
\mathbf{u}_{a}^{\prime}=\frac{\sqrt{1-v^{2} / c^{2}} \mathbf{u}_{a}+\left(\mathbf{u}_{a} \cdot \mathbf{v}\right) \mathbf{v}\left[1-\sqrt{1-v^{2} / c^{2}}\right] / v^{2}-\mathbf{v}}{1-\mathbf{u}_{a} \cdot \mathbf{v} / c^{2}}
$$

where we assume that if an object is observed in one system, then this object also should be observed in another system, which is uniformly moving relative to the former such that Eqs. (8) and (9) are always meaningful. Moreover, we only consider here the case of $v<c$, i.e., bradyonic motion of the system of coordinates since it is remainly doubtful whether the physically acceptable Lorentz transformation for the case of $v>c$ exists or not [16]. Most importantly, the sign-factor of Eq. (8), which has no effect for the usual treatment of the bradyon, is now the novel effect of the tachyon. Then, using an identity from Eq. (8)

$$
1=\gamma \frac{\sqrt{1-u_{a}^{\prime 2} / c^{2}}}{\sqrt{1-u_{a}^{2} / c^{2}}}\left\{1-\frac{\mathbf{u}_{a} \cdot \mathbf{v}}{c^{2}}\right\} \operatorname{sign}\left\{1-\frac{\mathbf{u}_{a} \cdot \mathbf{v}}{c^{2}}\right\},
$$

where $\gamma=1 / \sqrt{1-v^{2} / c^{2}}$, and Eq. (9), Eqs. (6) and (7) can be rewritten as follows

$$
\begin{aligned}
\sum_{a} m_{a}^{\prime}= & \sum_{a} m_{a}^{\prime}\left[\gamma \frac{\sqrt{1-u_{a}^{\prime 2} / c^{2}}}{\sqrt{1-u_{a}^{2} / c^{2}}}\left\{1-\frac{\mathbf{u}_{a} \cdot \mathbf{v}}{c^{2}}\right\} \operatorname{sign}\left\{1-\frac{\mathbf{u}_{a} \cdot \mathbf{v}}{c^{2}}\right\}\right] \\
= & \gamma\left[\sum_{a} m_{a}-\frac{\mathbf{v}}{c^{2}} \cdot \sum_{a}\left(m_{a} \mathbf{u}_{a}\right)\right], \\
\sum_{a}\left(m_{a}^{\prime} \mathbf{u}_{a}^{\prime}\right)= & \sum_{a} m_{a}^{\prime}\left[\frac{\sqrt{1-u_{a}^{\prime 2} / c^{2}}}{\sqrt{1-u_{a}^{2} / c^{2}}}\left\{\mathbf{u}_{a}+(\gamma-1) \frac{\left(\mathbf{u}_{a} \cdot \mathbf{v}\right) \mathbf{v}}{v^{2}}-\gamma \mathbf{v}\right\}\right. \\
& \left.\times \operatorname{sign}\left\{1-\frac{\mathbf{u}_{a} \cdot \mathbf{v}}{c^{2}}\right\}\right] \\
= & {\left[1+(\gamma-1) \frac{\mathbf{v}}{v^{2}} \mathbf{v} \cdot\right] \sum_{a}\left(m_{a} \mathbf{u}_{a}\right)-\gamma \mathbf{v} \sum_{a} m_{a}, }
\end{aligned}
$$

where

$$
m_{a}=m_{a}^{\prime} \frac{\sqrt{1-u_{a}^{\prime 2} / c^{2}}}{\sqrt{1-u_{a}^{2} / c^{2}}} \operatorname{sign}\left\{1-\frac{\mathbf{u}_{a} \cdot \mathbf{v}}{c^{2}}\right\} .
$$

Note that the transformation laws (10) and (11) are uniquely determined by applying our definition of the mass, i.e., if $\sum_{a} m_{a}$ and $\sum_{a} m_{a} \mathbf{u}_{a}$ are conserved in $S$, this should be also true for $\sum_{a} m_{a}^{\prime}$ and $\sum_{a} m_{a}^{\prime} \mathbf{u}_{a}^{\prime}$ in $S^{\prime}$. Hence, the mass transformation law (12) is unique. Without the 
sign factor in Eq. (12), Eqs. (10) and (11) show that the conservation of $\sum_{a} m_{a}$ and $\sum_{a} m_{a} \mathbf{u}_{a}$ does not always imply the conservation of $\sum_{a} m_{a}^{\prime}$ and $\sum_{a} m_{a}^{\prime} \mathbf{u}_{a}^{\prime}$.

Now, it remains to obtain the mass function $m$ satisfying the mass transformation (12) as a function of dynamical variables. To this end, we first consider the case of the bradyon, i.e., $u_{a}^{2}<c^{2}$ or equivalently $u_{a}^{\prime 2}<c^{2}$. In this case, the mass transformation (12) is reduced to be

$$
m_{a}=m_{a}^{\prime} \frac{\sqrt{1-u_{a}^{\prime 2} / c^{2}}}{\sqrt{1-u_{a}^{2} / c^{2}}}
$$

or

$$
m_{a} \sqrt{1-u_{a}^{2} / c^{2}}=m_{a}^{\prime} \sqrt{1-u_{a}^{\prime 2} / c^{2}}=\text { frame independent constant. }
$$

By considering especially the object rest system with $u_{a}=0$, we can see that the frame independent constant, which should be an inherent property of the object, is nothing but the object's mass when it is at rest, i.e., rest mass $m_{0}$. Then, the mass for the non-zero velocity objects can be obtained as

$$
m_{a}\left(\mathbf{u}_{a}\right)=\frac{m_{0 a}}{\sqrt{1-u_{a}^{2} / c^{2}}} .
$$

Note that this does not depend on the direction of the objects' velocity, but only it's magnitude. Actually this property can be easily understood using the isotropy of the space. Suppose that there is a rest object in a system of coordinates $S$. Then, if there is the mass relation between the rest object and the object moving relative to the rest one for the same kind objects, the transformation function should not depend on the direction of the velocity of the moving object since all the directions relative to the rest object are equivalent due to the isotropy of the space (Fig.1).

On the other hand, the situation is very different for the case of tachyon, i.e., $u_{a}^{2}>c^{2}$ or equivalently $u_{a}^{\prime 2}>c^{2}$. In this case the anomalous sign-factor in the mass transformation (12) becomes important. Due to this novel sign-factor, the quantity $m_{a} \sqrt{1-u_{a}^{2} / c^{2}}$ is no more the frame independent one. Rather the true frame independent quantity is the squared quantity of this, i.e.,

$$
m_{a}^{2}\left\{u_{a}^{2} / c^{2}-1\right\}=m_{a}^{\prime 2}\left\{u_{a}^{\prime 2} / c^{2}-1\right\}=\text { frame independent constant. }
$$

Now, by considering the system of coordinates when the object's velocity is $u_{a}^{2}=2 c^{2}$, we can see that the frame independent constant, which should be also an inherent property of the tachyonic object, is nothing but the square of the objects' mass at $u_{a}^{2}=2 c^{2}$. Then the square of the mass for the general velocity of the tachyon is determined as

$$
m_{a}^{2}\left(\mathbf{u}_{a}\right)=\frac{\text { square of mass at } u_{a}^{2}=2 c^{2}}{u_{a}^{2} / c^{2}-1} .
$$


But this formula does not determine the sign of the mass, i.e., when the mass function is expressed, without destroying the generality as follows

$$
m_{a}=\frac{\kappa_{a}}{\sqrt{u_{a}^{2} / c^{2}-1}}
$$

the sign of $\kappa_{a}$, which may depend on it's motion, is not determined but only $\kappa_{a}^{2}$ is found to be frame independent.

Before we discuss the determination of the sign-factor, it seems appropriate to show that the mass function $m_{a}$ and hence $\kappa_{a}$ may depend on the direction of the velocity in contrast to the case of the bradyon. This can be easily understood by the fact that any tachyon's mass is determined by comparing with the mass of the object with the same kinds having the velocity $u_{a}^{2}=2 c^{2}$ and a definite direction. Hence, all the directions of the velocity of the general tachyon are not equivalent due to the asymmetry of the situation. So if there is any relation between the mass of the general tachyon with that of the tachyon of $u_{a}^{2}=2 c^{2}$, the function can be depend on the velocity of the tachyon relative to the tachyon of $u_{a}^{2}=2 c^{2}$ in general (Fig. 2).

Now we discuss on the sign problem of the mass. To this end we first note that $\kappa_{a}$, which is called the proper mass of the tachyon, can be expressed as

$$
\kappa_{a}=\left|\kappa_{a}\right| \cdot(\text { sign-function })
$$

where the magnitude of $\kappa,\left|\kappa_{a}\right|$, does not depend on the direction of the tachyon's velocity since $\kappa_{a}^{2}$ is the frame independent constant, and hence only the "sign-function" may depend on the direction of the velocity as guaranteed by the asymmetry of the situation of the mass determination method. As for the sign-function, we first note that the tachyon's mass and hence the sign-function do not depend on the direction of the tachyon for a given speed. This can be understood by considering the collision process of the tachyon with the bradyon. If the mass of the tachyon depends on the direction of the velocity for a given speed, it should also be changed for the different orientations of the coordinates (Fig. 3). But, this is not allowed in order that the total energy and momentum of Eq.(4) and (5) are conserved in any orientation of the coordinates because the bradyon's mass is independent on the direction of the velocity. Hence, the tachyon's mass has a definite sign for a given speed in a uniformly moving system. However, the sign itself can not be determined a priori but only by actual measurement for a given situation. For the method of determination of the mass, we can imagine that the sign is uniquely determined by considering the collision-process between the tachyons having the given speed, with the bradyon operationally since the bradyon has a definite sign of the mass for any motions. However, for the different velocity the sign of the mass can be changed depending on the direction and the magnitude of the tachyon's velocity relative to the relative motion of the 
system of coordinates according to Eq. (12). In the expression of the tachyon's proper mass $\kappa_{a}$, the Eq. (12) implies

$$
\kappa_{a}=\kappa_{a}^{\prime} \operatorname{sign}\left\{1-\frac{\mathbf{u}_{a} \cdot \mathbf{v}}{c^{2}}\right\}
$$

since the mass in the system $S^{\prime}$ also should be expressed as

$$
m_{a}^{\prime}=\frac{\kappa_{a}^{\prime}}{\sqrt{u_{a}^{\prime 2} / c^{2}-1}}
$$

due to the principle of relativity. Hence the proper mass of the tachyon is not an absolute quantity in contrast to that of the bradyon. This is the novel feature of the tachyon. Furthermore, we note that although the sign-factor of Eq. (20) is ill-defined for the case of $1-\mathbf{u} \cdot \mathbf{v} / c^{2}=0$, i.e., $u_{a}^{\prime}=\infty$ from Eq. (8) or (9) such that the sign of $\kappa_{a}^{\prime}$ is ill-defined in this case, the directly measurable quantity $m_{a}^{\prime}$ is well-defined according to Eq. (21): $m_{a}^{\prime}$ becomes zero in this case for any finite $\kappa_{a}^{\prime}$, which is expected to be the only physically realizable case.

\section{B. Energy-momentum transformation law and it's implications}

We define momentum as the quantity

$$
\mathbf{p}_{a}=m_{a}\left(\mathbf{u}_{a}\right) \mathbf{u}_{a}
$$

which is conserved through all possible process of collision and in all systems of coordinates for the $a$ 'th object.

Moreover, we fundamentally define the (kinetic) energy $E_{a}$ of the object such that the change of the mass of the object between one state of motion $A$ and another state of motion $B$ is completely spent to change the energy of the object, i.e.,

$$
m_{a}(B) c^{2}-m_{a}(A) c^{2}=E_{a(B)}-E_{a(A)}
$$

Then, the energy has the expression as

$$
E_{a}=m_{a}\left(\mathbf{u}_{a}\right) c^{2}+\epsilon_{a}
$$

where $\epsilon_{a}$ is the integration constant, which is inherent to the object and hence may be different for the different species of object generally, and is related to the choice of the origin of the energy such that it may be expected that they have arbitrary values [4]. However, by defining the energy also as the conserved quantity for all the possible processes of collision and all the 
systems of coordinates, we can obtain some constraints about the value of $\epsilon_{a}$. To this end, let us first note that Eq. (11) can be rewritten as

$$
\begin{aligned}
\sum_{a}\left(m_{a}^{\prime} \mathbf{u}_{a}^{\prime}\right)= & {\left[1+(\gamma-1) \frac{\mathbf{v}}{v^{2}} \mathbf{v} \cdot\right] \sum_{a}\left(m_{a} \mathbf{u}_{a}\right) } \\
& -\gamma \frac{\mathbf{v}}{c^{2}} \sum_{a}\left(m_{a} c^{2}+\epsilon_{a}\right)+\gamma \frac{\mathbf{v}}{c^{2}} \sum_{a} \epsilon_{a}
\end{aligned}
$$

or

$$
\begin{aligned}
\sum_{a} \mathbf{p}_{a}^{\prime}= & {\left[1+(\gamma-1) \frac{\mathbf{v}}{v^{2}} \mathbf{v} \cdot\right] \sum_{a} \mathbf{p}_{a} } \\
& -\gamma \frac{\mathbf{v}}{c^{2}} \sum_{a} E_{a}+\gamma \frac{\mathbf{v}}{c^{2}} \sum_{a} \epsilon_{a} .
\end{aligned}
$$

Now, in order that through the process of collision the total momentum is conserved in $S^{\prime}$, we must also have the conservation of $\sum_{a} \epsilon_{a}$, i.e.,

$$
\sum_{a_{i}=1}^{M} \epsilon_{a_{i}}=\sum_{a_{f}=1}^{N} \epsilon_{a_{f}}
$$

for the In state $i$ and Out state $f$, as well as the total energy and momentum in $S$ (Fig. 4). Here it is easy to see, by considering the pair-creation processes, that $\epsilon_{a}=0$ for the strictly neutral particles, which are defined as the object having the property of " particle $=$ antiparticle " [17], but $\epsilon_{a}$ (particle) $=-\epsilon_{a}$ (antiparticle) in the other cases. In the latter cases, the value of $\epsilon_{a}$ itself is remainly undetermined. Furthermore, note that this is essentially the relativistic effect, which has not been known before: the last term in Eq. (26), which is crucial to this effect, does not appear in the non-relativistic limit of $v \rightarrow 0$.

Independently with this argument, we consider, now, the transformation law of Eq. (10). By noting that Eq. (10) can be reexpressed as

$$
\sum_{a}\left(m_{a}^{\prime} c^{2}+\epsilon_{a}^{\prime}\right)-\sum_{a} \epsilon_{a}^{\prime}=\gamma\left[\sum_{a}\left(m_{a} c^{2}+\epsilon_{a}\right)-\sum_{a} \epsilon_{a}-\mathbf{v} \cdot \sum_{a}\left(m_{a} \mathbf{u}_{a}\right)\right],
$$

we obtain the following relation

$$
\sum_{a} E_{a}^{\prime}-\sum_{a} \epsilon_{a}^{\prime}=\gamma\left[\sum_{a} E_{a}-\mathbf{v} \cdot \sum_{a} \mathbf{p}-\sum_{a} \epsilon_{a}\right]
$$

where

$$
E_{a}^{\prime}=m_{a}^{\prime} c^{2}+\epsilon_{a}^{\prime}
$$


Hence the total energy is also conserved in $S^{\prime}$ through the process of collision, when the total energy and momentum are conserved in $S$ only if the integration constant is also conserved in $S^{\prime}$ since we already know it's conservation in $S$ form Eq. (27). Due to this conservation of $\epsilon_{a}^{\prime}$ in $S^{\prime}$ the constraints on it's value described in the below Eq. (27) are universally valid in all systems of coordinates.

Furthermore, note that these results for the property of $\epsilon_{a}$ is valid for both the tachyon and the bradyon. It will be shown later in Section IV that these are also true for the case of the luxon.

When expressed with the total momentum and energy, Eqs. (26) and (29) with $\epsilon_{a} \equiv 0$ as in the usual treatment, now become

$$
\begin{aligned}
\mathbf{p}^{\prime} & =\left[1+(\gamma-1) \frac{\mathbf{v}}{v^{2}} \mathbf{v} \cdot\right] \mathbf{p}-\gamma \frac{\mathbf{v}}{c^{2}} E, \\
E^{\prime} & =\gamma[E-\mathbf{p} \cdot \mathbf{v}]
\end{aligned}
$$

where

$$
\begin{aligned}
\mathbf{p} & =\sum_{a} m_{a} \mathbf{u}_{a}, \quad E=\sum_{a} m_{a} c^{2}, \\
\mathbf{p}^{\prime} & =\sum_{a} m_{a}^{\prime} \mathbf{u}_{a}^{\prime}, \quad E^{\prime}=\sum_{a} m_{a}^{\prime} c^{2},
\end{aligned}
$$

and the single point object also satisfies the same transformation law. The transformation law (32) shows that the sign of the (total) energy may be changed when the (center of mass) velocity $\mathbf{u}$ is faster than that of light because

$$
E^{\prime}=\gamma E\left\{1-\frac{\mathbf{u} \cdot \mathbf{v}}{c^{2}}\right\}
$$

for the (center of mass) single object such that $\mathbf{p}=\left(E / c^{2}\right) \mathbf{u}$.

Although this has been known as the novel feature of the usual tachyon theory, but this is actually not the case, i.e., this phenomena can not be obtained in the usual formulation. To see this we note that in the usual formulation [2,8,9-11] the momentum and energy of the point objects are assumed to be

$$
\begin{aligned}
& \mathbf{p}=\frac{\mu \mathbf{u}}{\sqrt{u^{2} / c^{2}-1}}, \\
& E=\frac{\mu c^{2}}{\sqrt{u^{2} / c^{2}-1}},
\end{aligned}
$$

where $\mu$ is constant, and is the same for all system of coordinates. The latter point is essentially due to the fact that the formulas (35) and (36) are obtained from the usual formulas (1)-(3) of 
the bradyon by substituting simply $m \rightarrow i \mu$ such that $\mathbf{p}$ and $E$ are real-valued for the velocity faster than that of light. With the help of the formula (8) we can easily see that

$$
\begin{aligned}
& \mathbf{p}^{\prime}=\left\{\left[1+(\gamma-1) \frac{\mathbf{v}}{v^{2}} \mathbf{v} \cdot\right] \mathbf{p}-\gamma \frac{\mathbf{v}}{c^{2}} E\right\} \operatorname{sign}\left\{1-\frac{\mathbf{u} \cdot \mathbf{v}}{c^{2}}\right\}, \\
& E^{\prime}=\gamma(E-\mathbf{p} \cdot \mathbf{v}) \operatorname{sign}\left\{1-\frac{\mathbf{u} \cdot \mathbf{v}}{c^{2}}\right\} .
\end{aligned}
$$

Hence the energy for the single object case becomes

$$
E^{\prime}=\gamma E\left|1-\frac{\mathbf{u} \cdot \mathbf{v}}{c^{2}}\right|
$$

showing that the sign of the energy does not change for any motion of the tachyon. Therefore the usual belief that the so called reinterpretation principle for " the state of tachyon of negative energy and reversed time sequence " of BDSF is needed to avoid the problem of the negative energy simultaneously with the causality problem is misunderstanding due to their wrong formulas of the usual formulation for the tachyon. Hence the original motivation for the BDSF's reinterpretation principle, if it applies to our Nature, can not be found in the usual formulation but only in our new formulation of the tachyon. Furthermore, as it is clear from the formula (37), total energy and momentum may be non-conserved in one system of coordinates although conserved in the other systems within the usual formulation [18]. It follows from this result that the tachyon can not be represented by simple replacement $m \rightarrow i \mu$ in contrast to the usual belief.

In addition we would like to remark that although there is the anomalous sign-factor in the mass transformation formulas (12) or (21), $E^{2}-c^{2} p^{2}$ is Lorentz invariant, and (E,cp) forms a four - vector for the tachyon as well as the bradyon.

\section{LEAST ACTION APPROACH TO THE TACHYON DYNAMICS}

In the previous section we have considered the tachyon dynamics in the most elementary way. Now, we reconsider the topics of the previous section in a more mathematical framework, i.e., the least action principle approach, and the results of the previous section will be rederived in this formulation. It is well known that the relativistic dynamics of point bradyon can be elegantly described by the least action principle. Moreover, remarkably the formulation of the free bradyon can be uniquely described by considering some Lorentz invariant quantities and presumed properties of the free objects, i.e., the only dynamical variables should be the velocity $\mathbf{u}$ of these objects although the interactions with other fields of force are not uniquely defined unless more informations or restrictions are not given [19]. 
However, as it will be clear later, the applicability of the approach for the tachyon and luxon is not trivial matter. These problem will be treated in this and next section, where it will be shown that the least action principle is also applicable to the case of the tachyon and luxon.

\section{A. The world-parameter}

For the usual bradyonic object there is an invariant parameter $\tau$, which is called the proper time of this object, such that

$$
\begin{aligned}
c d \tau & \equiv c d t \sqrt{1-u^{2} / c^{2}}=c d t^{\prime} \sqrt{1-u^{\prime 2} / c^{2}} \\
& =c d \tau^{\prime}
\end{aligned}
$$

with the help of the usual velocity transformation of the bradyon and the parameter very useful to parameterize the world-trajectories of the relativistic point objects with the Lorentz invariant fashion. So we can expect that it would be also useful to introduce similar parameter for the world-trajectory of the tachyon in the formulation.

To this end, let us introduce the world-parameter $w$ describing the world-trajectories of the relativistic point objects including the tachyons as well as the bradyons unifiedly by the infinitesimal increment

$$
c d w \equiv d t \sqrt{d x^{\mu} d x_{\mu} /(d t)^{2}}=c d t \sqrt{1-u^{2} / c^{2}}
$$

for the infinitesimal interval in the world-trajectory of the object. Here we use $x^{\mu} \equiv(c t, \mathbf{x}), x_{\mu}=$ $\eta_{\mu \nu} x^{\nu}=(c t,-\mathbf{x})$, and $\eta_{\mu \nu}=\operatorname{diag}(+1,-1,-1,-1)$. By the general velocity transformation law (8) and the usual Lorentz transformation, which are also valid for tachyon as well as the bradyon, the transformation law of $c d w$ becomes

$$
\begin{aligned}
c d w^{\prime} & =c d t^{\prime} \sqrt{1-u^{\prime 2} / c^{2}} \\
& =c \gamma\left\{d t-\frac{\mathbf{v} \cdot d \mathbf{x}}{c^{2}}\right\} \frac{\sqrt{1-u^{2} / c^{2}}}{\gamma\left|1-\mathbf{u} \cdot \mathbf{v} / c^{2}\right|} \\
& =c d t \sqrt{1-u^{2} / c^{2}} \frac{\left\{1-\mathbf{u} \cdot \mathbf{v} / c^{2}\right\}}{\left|1-\mathbf{u} \cdot \mathbf{v} / c^{2}\right|} \\
& =\operatorname{sign}\left\{1-\frac{\mathbf{u} \cdot \mathbf{v}}{c^{2}}\right\} c d w .
\end{aligned}
$$

Therefore, the world-parameter transforms as

$$
w^{\prime}=\operatorname{sign}\left\{1-\frac{\mathbf{u} \cdot \mathbf{v}}{c^{2}}\right\} w
$$


by choosing appropriate origins of $w$ and $w^{\prime}$ using the homogeneity of the time $t$. Note that $w$ is not Lorentz invariant any more, but a pseudo-Lorentz invariant, i.e., Lorentz invariant up to a sign-factor [20], while $w^{2}$ is the Lorentz invariant as in the usual case. Especially, for bradyonic case the world-parameter $w$ itself becomes to be Lorentz invariant for any uniform motion of the system, and is reduced to the usual proper time $\tau$, i.e., the time measured by a clock which being rest at the objects. Although this interpretation is not possible for tachyon case, but we can say that $w$ has the same value of the time (interval) of the tachyon measured by a clock (of course, being made of bradyons) whose velocity is $\sqrt{2} c$ relative to the tachyon. Moreover, in this case if we introduce the real valued world-parameter $\lambda$ by $\lambda \equiv-i w$ such that

$$
c d \lambda=c d t \sqrt{u^{2} / c^{2}-1}
$$

then $\lambda$ transforms as

$$
\lambda^{\prime}=\operatorname{sign}\left\{1-\frac{\mathbf{u} \cdot \mathbf{v}}{c^{2}}\right\} \lambda,
$$

where the anomalous sign factor can not be neglected. Here we note that the real parameters $\tau$ and $\lambda$ can be used to parameterize the length of the world trajectories of bradyon and tachyon, respectively.

Before ending this subsection it seems appropriate to comment on our choice of the worldparameter as Eq. (40). In the usual formulation of the relativistic point bradyon, the two definitions

$$
c d \tau \equiv d t \sqrt{\left(d x^{\mu} d x_{\mu}\right) /(d t)^{2}}=c d t \sqrt{1-u^{2} / c^{2}}
$$

and

$$
\begin{aligned}
c d \hat{\tau} & \equiv \sqrt{d x^{\mu} d x_{\mu}}=|d t| \sqrt{\left(d x^{\mu} d x_{\mu}\right) /(d t)^{2}} \\
& =c|d t| \sqrt{1-u^{2} / c^{2}}
\end{aligned}
$$

are interchangeably used. This is essentially due to the fact that, for the case of the bradyon the sign of $d t$ and hence the order of events are Lorentz invariant. Due to this fact, both $\tau$ and $\hat{\tau}$ equally describe the trajectory invariantly. Note that the time direction of the world-parameter can be chosen to be the same as that of $t$ for $\tau$, or always positive for both the positive and negative direction of $t$ for $\hat{\tau}$, and moreover this assignment of the sign is Lorentz invariant. Hence, in this case the two parameters have the equivalent role. Mathematically, this fact can be expressed by the fact that both $\tau$ and $\hat{\tau}$ are Lorentz invariant [19].

However, this situation is drastically changed for the case of the tachyon. In this case $w$ in Eq. (40) and other definition similar to Eq. (46) as

$$
\begin{aligned}
c d \hat{w} & \equiv \sqrt{d x^{\mu} d x_{\mu}}=|d t| \sqrt{\left(d x^{\mu} d x_{\mu}\right) /(d t)^{2}} \\
& =c|d t| \sqrt{1-u^{2} / c^{2}}
\end{aligned}
$$


have different role each other, and so can not be used interchangeably. Since the sign of $d t$ for the tachyon is not Lorentz invariant, $w$ is not Lorentz invariant although $\hat{w}$ is. However, essentially because of this fact, $\hat{w}$ can not be used as the world-parameter for the tachyon. Note that since $\hat{w}$ is blind for the time reversion, which is always possible for the tachyon, $\hat{w}$ can not describe the time reversion phenomena of the tachyon, but $w$ is not the case. So we can conclude that the definitions (40) and (45) are more fundamental than those of (46) and (47). This is the reason why we does not introduce the Lorentz invariant parameter $\hat{w}$ as the worldparameter but introduce the parameter $w$, which is not Lorentz invariant, as in Eq. (40). This situation is comparative to that for the classical theory of the pair-creation or annihilation of a particle and it's anti-particle of Stüeckelberg and Feynman [21], where $\hat{\tau}$ is more favored than $\tau$.

\section{B. The world-velocity and acceleration}

Now, using the world-parameter $w$ we can define the world-velocity $\mathcal{U}^{\mu}$ describing the tachyon as well as the bradyon unifiedly, as follows

$$
\mathcal{U}^{\mu} \equiv \frac{d x^{\mu}}{d w}
$$

Then, due to the four-vector property of the quantity $d x^{\mu}$ and the pseudo-scalar property of $d w$, the world-velocity $\mathcal{U}^{\mu}$ becomes the quantity of the pseudo four-vector, and it's components are given by

$$
\begin{aligned}
\mathcal{U}^{\mu} & =\left(\frac{c d t}{d w}, \frac{d \mathbf{x}}{d w}\right) \\
& =\left(\frac{c}{\sqrt{1-u^{2} / c^{2}}}, \frac{\mathbf{u}}{\sqrt{1-u^{2} / c^{2}}}\right),
\end{aligned}
$$

where $\mathbf{u} \equiv d \mathbf{x} / d t$. This transforms under the Lorentz transformation as

$$
\begin{aligned}
\mathcal{U}^{\prime \mu} & =\left(\frac{c d t^{\prime}}{d \omega^{\prime}}, \frac{d \mathbf{x}^{\prime}}{d \omega^{\prime}}\right) \\
& =\frac{1}{\operatorname{sign}\left\{1-\mathbf{u} \cdot \mathbf{v} / c^{2}\right\}} \Lambda_{\nu}^{\mu}\left(\frac{c d t}{d \omega}, \frac{d \mathbf{x}}{d \omega}\right) \\
& =\frac{1}{\operatorname{sign}\left\{1-\mathbf{u} \cdot \mathbf{v} / c^{2}\right\}} \Lambda^{\mu}{ }_{\nu} \mathcal{U}^{\nu},
\end{aligned}
$$


where $\Lambda_{\nu}^{\mu}$ is the general Lorentz transformation matrix, for the velocity $\mathbf{v}$ of the system $S^{\prime}$ relative to the system $S$, without rotation as

$$
d x^{\prime \mu}=\Lambda_{\nu}^{\mu} d x^{\nu}
$$

with components

$$
\begin{aligned}
\Lambda_{0}^{0} & =1 / \sqrt{1-v^{2} / c^{2}}, \\
\Lambda_{0}^{i} & =\Lambda_{i}^{0}=\left(v^{i} / c\right) / \sqrt{1-v^{2} / c^{2}}, \\
\Lambda_{j}^{i} & =\delta_{i j}+v^{i} v^{j} \frac{\left(1 / \sqrt{1-v^{2} / c^{2}}-1\right)}{v^{2}} .
\end{aligned}
$$

For the bradyon, the world-velocity $\mathcal{U}^{\mu}$ becomes the usual velocity four-vector $u^{\mu}(\tau)$, which is tangential to the trajectory with increasing $\tau$. In this case, $u^{\mu}$ is time-like, i.e., $u^{\mu} u_{\mu}=c^{2}>0$. On the other hand, the corresponding quantity $t^{\mu}$ for the tachyon, which is real-valued, being defined as follows

$$
\begin{aligned}
t^{\mu} \equiv i \mathcal{U}^{\mu} & =\left(\frac{c d t}{d \lambda}, \frac{d \mathbf{x}}{d \lambda}\right) \\
& =\left(\frac{c}{\sqrt{u^{2} / c^{2}-1}}, \frac{\mathbf{u}}{\sqrt{u^{2} / c^{2}-1}}\right)
\end{aligned}
$$

is the tangential to the world trajectory with increasing $\lambda$, and is space-like, i.e., $t^{\mu} t_{\mu}=-c^{2}<0$. The transformation laws for these two distinct cases are given by

$$
\begin{aligned}
u^{\prime \mu} & =\Lambda_{\nu}^{\mu} u^{\nu}, \\
t^{\prime \mu} & =\frac{1}{\operatorname{sign}\left\{1-\mathbf{u} \cdot \mathbf{v} / c^{2}\right\}} \Lambda_{\nu}^{\mu} t^{\nu} .
\end{aligned}
$$

As an another independent kinematic world-quantity, we introduce world-acceleration vector $\mathcal{A}^{\mu}$ as follows

$$
\mathcal{A}^{\mu} \equiv \frac{d \mathcal{U}^{\mu}}{d w}
$$

Then due to the pseudo-vector property of $\mathcal{U}^{\mu}$ and pseudo-scalar property of $w$, the world acceleration $\mathcal{A}^{\mu}$ becomes the four-vector, i.e, it transforms the same as $d x^{\mu}$ under the Lorentz transformation, as usual. It's components are given by

$$
\begin{aligned}
\mathcal{A}^{\mu} & =\left(\frac{c d^{2} t}{d w^{2}}, \frac{d^{2} \mathbf{x}}{d w^{2}}\right) \\
& =\left(\frac{\mathbf{u} \cdot \mathbf{a}}{c\left(1-u^{2} / c^{2}\right)^{2}}, \frac{\mathbf{a}}{1-u^{2} / c^{2}}+\frac{(\mathbf{u} \cdot \mathbf{a}) \mathbf{u}}{c^{2}\left(1-u^{2} / c^{2}\right)^{2}}\right)
\end{aligned}
$$


with the square magnitude of the four-vector

$$
\begin{aligned}
\mathcal{A}^{\mu} \mathcal{A}_{\mu} & =-\frac{a^{2} c^{2}+(\mathbf{a} \cdot \mathbf{u})^{2}-a^{2} u^{2}}{c^{2}\left(1-u^{2} / c^{2}\right)^{3}} \\
& =-\frac{\left\{a^{2} c^{2}-a^{2} u^{2} \sin ^{2} \phi\right\}}{c^{2}\left(1-u^{2} / c^{2}\right)^{3}}
\end{aligned}
$$

where $\phi$ is the angle between the three-velocity vector $\mathbf{u}$ and three-acceleration vector $\mathbf{a}=$ $d \mathbf{u} / d t$. And this world-acceleration vector $\mathcal{A}^{\mu}$ is the vector which is normal to the tangential vector $\mathcal{U}_{\mu}$, i.e.,

$$
\mathcal{U}_{\mu} \mathcal{A}^{\mu}=\mathcal{U}_{\mu} \frac{d \mathcal{U}^{\mu}}{d w}=0
$$

from the relation $\mathcal{U}_{\mu}\left(d \mathcal{U}^{\mu} / d w\right)=(1 / 2) d\left(\mathcal{U}_{\mu} \mathcal{U}^{\mu}\right) / d w$ and

$$
\mathcal{U}_{\mu} \mathcal{U}^{\mu}=c^{2} .
$$

For the bradyon, the world-acceleration vector coincides with the usual four-acceleration vector $a^{\mu}$ as

$$
\mathcal{A}^{\mu}=\frac{d \mathcal{U}^{\mu}}{d w}=\frac{d u^{\mu}}{d \tau} \equiv a^{\mu}
$$

because $\mathcal{U}^{\mu}=u^{\mu}$ and $w=\tau$ in this case. The square of the $a^{\mu}$ is always negative definite due to the two facts:

$$
\begin{aligned}
& \text { i) } a^{2} c^{2}-a^{2} u^{2} \sin ^{2} \phi \geq a^{2} c^{2}-a^{2} u^{2}>0, \\
& \text { ii) }\left(1-u^{2} / c^{2}\right)^{3}>0
\end{aligned}
$$

,i.e., $a^{\mu}$ is the space-like vector (Fig. 5).

On the other hand, for the tachyon, an interesting new phenomenon occurs. In this case with the reasonable definition of the acceleration vector

$$
a_{t}^{\mu} \equiv \frac{d t^{\mu}}{d \lambda}=-\frac{d \mathcal{U}^{\mu}}{d w}=-\mathcal{A}^{\mu}
$$

since $t^{\mu}=i \mathcal{U}^{\mu}$ and $\lambda=-i w$ in this case, the norm of $a_{t}^{\mu}$ is not always negative value due to the two facts:

$$
\begin{aligned}
& \text { i) } \quad a^{2} c^{2}-a^{2} u^{2} \sin ^{2} \phi \frac{\leq}{>} 0 \text { depending on } \sin ^{2} \phi \frac{<}{>} \frac{c^{2}}{u^{2}} \\
& \text { ii) } \quad\left(1-u^{2} / c^{2}\right)^{3}<0 .
\end{aligned}
$$


Rather $a_{t}^{\mu}$ is space-like or time-like or light-like depending on the value of angle $\phi$ for a given velocity u (Fig. 6).

\section{Least action approach to the tachyon dynamics}

In the least action approach one fundamentally assume that one can define a world-scalar action by which we obtain the covariant equations of motion of the interested objects by applying an invariant principle, i.e., the least action principle. Therefore, it is essential to find the Lorentz invariant combination called the action, which is appropriate for the system considered, or introduce some new parameters and variables such that the action is Lorentz invariant to proceed this approach if there is no non-trivial form of the Lorentz invariant action with the given dynamical variables.

Now, since for the free objects the only dynamical variables are $\mathcal{U}^{\mu}$, the possible candidate for the invariant combinations are $\mathcal{U}^{\mu} \mathcal{U}_{\mu},\left(\mathcal{U}^{\mu} \mathcal{U}_{\mu}\right)^{2}, \ldots$, etc. . However, unfortunately only these terms are not appropriate for the description of the dynamics because these terms are nothing but the power of non-dynamical universal constant $c$ from the relation (59). Since these are all the possible Lorentz invariant combinations from the dynamical variable $\mathcal{U}^{\mu}$, we must consider the other possibilities to construct the Lorentz invariant action. To this end we first consider the world-parameter $w$. However, since this is not Lorentz invariant but pseudo-invariant, we must introduce some pseudo-invariant coefficient to cancel out the additional sign-factor which comes from the transformation law of $w$, and hence make their product Lorentz invariant.

Let us define the Lorentz invariant world-action $\mathcal{J}$ describing the tachyon as well as the bradyon formally, as

$$
\mathcal{J}\left[w_{1}, w_{2}\right]=-\int_{w_{1}}^{w_{2}} \mathcal{U}^{\mu} \mathcal{U}_{\mu} K d w=-c^{2} \int_{w_{1}}^{w_{2}} K d w
$$

where $K$ has the mass dimension, and has pseudo-invariant property under the Lorentz transformations (51) and (52), i.e.,

$$
K^{\prime}=\operatorname{sign}\left\{1-\frac{\mathbf{u} \cdot \mathbf{v}}{c^{2}}\right\} K
$$

such that the product of $K$ and $d w$ is Lorentz invariant. We call $K$ as " the world-mass parameter". Moreover, note that the combination $\int \mathcal{U}^{\mu} d x_{\mu}$ reduces also to the form of (64). This is our fundamental assumption of the least action approach to the general dynamics of the free objects with the tachyon as well as the bradyon. When expressed by the three-velocity $\mathbf{u}$ with the relation (40), the world-action becomes

$$
\mathcal{J}=-c^{2} \int_{t_{1}}^{t_{2}} K \sqrt{1-u^{2} / c^{2}} d t
$$


which explicitly shows the reparametrization invariance $t \rightarrow \tilde{t}(t)$ for the monotonically increasing function $\tilde{t}(t)$ such that

$$
\left|\frac{d \tilde{t}}{d t}\right|=\frac{d \tilde{t}}{d t}
$$

is satisfied for all the time $t$. With the help of the reparametrization invariance the least action principle can be also applied to the tachyon without any ambiguity as well as the bradyon. For the bradyon, the usual (virtual) variational method to derive the equations of motion with the fixed time $t$ is well defined for any bradyonic trajectory since the time interval $\Delta t=t_{A}-t_{B}$ for any two events $A$ and $B$ along the world-line of the object is always non-zero such that the derivatives by " $d t$ " have no ambiguities.

By defining the Lagrangian $L(t)$ for the bradyon with the physical time $t$ as

$$
\mathcal{J}\left[t_{1}, t_{2}\right]=\int_{t_{1}}^{t_{2}} L(t) d t
$$

with

$$
L(t)=-m_{0} c^{2} \sqrt{1-u^{2} / c^{2}}
$$

and the canonical linear momentum $\mathbf{p}(t)$ as

$$
\mathbf{p}(t) \equiv \frac{\partial L(t)}{\partial(d \mathbf{x} / d t)}=\frac{\partial L(t)}{\partial \mathbf{u}}=\frac{m_{0} \mathbf{u}}{\sqrt{1-u^{2} / c^{2}}},
$$

the equation of motion becomes

$$
\frac{d \mathbf{p}}{d t}=0
$$

from the least action condition

$$
\begin{aligned}
0=\delta \mathcal{J}\left(t_{1}, t_{2}\right) & =\int_{t_{1}}^{t_{2}} \delta L(t) d t \\
& =\int_{t_{1}}^{t_{2}}\left[\frac{\partial L}{\partial \mathbf{x}}-\frac{d}{d t}\left(\frac{\partial L}{\partial(d \mathbf{x} / d t)}\right)\right] \cdot \delta \mathbf{x} d t
\end{aligned}
$$

with the auxiliary condition

$$
\delta \mathbf{x}\left(t_{1}\right)=\delta \mathbf{x}\left(t_{2}\right)=0
$$

under the fixed time (virtual) variation (Fig. 7). Here we designate the world-mass parameter by the Lorentz invariant mass $m_{0}$ since in this case $K$ is not the pseudo-scalar any more but only a Lorentz scalar. The corresponding Hamiltonian becomes

$$
H(t) \equiv \mathbf{p} \cdot \mathbf{u}-L=\frac{m_{0} c^{2}}{\sqrt{1-u^{2} / c^{2}}},
$$


which is also constant in time for the system when

$$
\frac{\partial L}{\partial t}=0
$$

is satisfied, which is actually the case of ours. Furthermore, since the action (68) and the least action principle (72) are Lorentz invariant, the conservation law of the momentum $\mathbf{p}$ and energy $E$ are also satisfied in all uniformly moving systems as in the case of the previous section. For many objects case, the conserved ones are found to be the total momentum and energy.

On the other hand, for the tachyon this approach is more involved, i.e., the usual variational method to derive the equations of motion with fixed time $t$ has some problem in this case. This is essentially due to the time interval $\Delta t=t_{B}-t_{A}$ between two events $A$ (leaving of the tachyon) and $B$ (arriving of the tachyon) along the world-line of the object always vanish for the infinite velocity tachyon, which is called transient tachyon [8], such that there are some ambiguities in deriving the equation of motion. Furthermore, since any tachyon can be made to have the infinite velocity by choosing an appropriate system of coordinates, it seems that this problem can not be avoided for any tachyon (Fig. 8). However, this problem can be overcome by using the reparametrization invariance.

To see this, we note that the world-action (66) parameterized by the tachyon's worldparameter $\lambda$ of Eqs. (43) and (44) becomes

$$
\begin{aligned}
\mathcal{J} & =c^{2} \int_{t_{1}}^{t_{2}} \kappa \sqrt{\frac{1}{c^{2}}\left(\frac{d \mathbf{x}}{d t}\right)^{2}-1} d t \\
& =c^{2} \int_{t_{1}}^{t_{2}} \kappa \sqrt{\frac{1}{c^{2}}\left(\frac{d \mathbf{x}}{d \lambda}\right)^{2}-\left(\frac{d t}{d \lambda}\right)^{2}}\left|\frac{d \lambda}{d t}\right| d t \\
& =c^{2} \int_{\lambda_{1}}^{\lambda_{2}} \kappa \sqrt{\frac{1}{c^{2}}\left(\frac{d \mathbf{x}}{d \lambda}\right)^{2}-\left(\frac{d t}{d \lambda}\right)^{2}} d \lambda
\end{aligned}
$$

where we have introduced the mass parameter $\kappa \equiv-i K$, which should be real-valued in order that the action is real-valued [22], and has the same transformation law as that of $K$, i.e.,

$$
\kappa^{\prime}=\operatorname{sign}\left\{1-\frac{\mathbf{u} \cdot \mathbf{v}}{c^{2}}\right\} \kappa .
$$

Here, we have used the fact [23]

$$
\frac{d \lambda}{d t}=\sqrt{u^{2} / c^{2}-1}>0
$$


Now, in this parameterization there is no singular case of $\Delta \lambda \equiv 0$ because $\Delta \lambda$ has an invariant magnitude and $\Delta \lambda$ is non-zero for any tachyon, including the transient tachyon as well, for any two different points along the world-line. The singular point of $\Delta t$ at $u=\infty$ is transferred to the singular point of $\Delta \lambda$ at $u=c$, i.e., the case of luxon such that there is no problem for the tachyon only case. Now, with the help of this property, the least action principle can be also applied in this case by adopting the fixed $\lambda$ (virtual) variations (Fig. 9) without any ambiguity .

By defining the Lagrangian $L(\lambda)$ with the parameter $\lambda$ as

$$
\mathcal{J}\left[\lambda_{1}, \lambda_{2}\right]=\int_{\lambda_{1}}^{\lambda_{2}} L(\lambda) d \lambda
$$

with

$$
L(\lambda)=\kappa c^{2} \sqrt{\frac{1}{c^{2}}\left(\frac{d \mathbf{x}}{d \lambda}\right)^{2}-\left(\frac{d t}{d \lambda}\right)^{2}} d \lambda,
$$

the equations of motion become

$$
\begin{aligned}
& 0=\frac{\partial L}{\partial t}-\frac{d}{d \lambda}\left[\frac{\partial L}{\partial(d t / d \lambda)}\right]=-\frac{d}{d \lambda}\left[\frac{\partial L}{\partial(d t / d \lambda)}\right] \\
& 0=\frac{\partial L}{\partial \mathbf{x}}-\frac{d}{d \lambda}\left[\frac{\partial L}{\partial(d \mathbf{x} / d \lambda)}\right]=-\frac{d}{d \lambda}\left[\frac{\partial L}{\partial(d \mathbf{x} / d \lambda)}\right]
\end{aligned}
$$

from the least action condition

$$
\begin{aligned}
0 & =\delta_{[\lambda]} \mathcal{J}\left[\lambda_{1}, \lambda_{2}\right]=\int_{\lambda_{1}}^{\lambda_{2}} \delta_{[\lambda]} L(\lambda) d \lambda \\
& =\int_{\lambda_{1}}^{\lambda_{2}}\left\{\left[\frac{\partial L}{\partial t}-\frac{d}{d \lambda}\left[\frac{\partial L}{\partial(d t / d \lambda)}\right]\right] \delta_{[\lambda]} t+\left[\frac{\partial L}{\partial \mathbf{x}}-\frac{d}{d \lambda}\left[\frac{\partial L}{\partial(d \mathbf{x} / d \lambda)}\right]\right] \cdot \delta_{[\lambda]} \mathbf{x}\right\} d \lambda
\end{aligned}
$$

with the auxiliary conditions

$$
\delta_{[\lambda]} x^{\mu}\left(\lambda_{1}\right)=\delta_{[\lambda]} x^{\mu}\left(\lambda_{2}\right)=0,
$$

and where $\delta_{[\lambda]}$ represents the variation with fixed $\lambda$. In this case the quantity

$$
\Pi(\lambda) \equiv \frac{\partial L}{\partial(d \mathbf{x} / d \lambda)}=\frac{\kappa(d \mathbf{x} / d \lambda)}{\sqrt{(d \mathbf{x} / c d \lambda)^{2}-(d t / d \lambda)^{2}}}
$$

can be considered as the canonical momentum, which is conserved with respects to the evolution of parameter $\lambda$ in all the uniformly moving systems because the conservation law (81) is satisfied 
in all uniformly moving systems due to the invariance of the world-action (79) and the least action principle (83). When expressed in the three-vector $\mathbf{u}, \boldsymbol{\Pi}(\lambda)$ becomes

$$
\boldsymbol{\Pi}(\lambda)=\frac{\kappa \mathbf{u}}{\sqrt{u^{2} / c^{2}-1}} \equiv \mathbf{p}(t)
$$

which is formally the same result as in the previous section.

Furthermore, the quantity

$$
-\Pi^{0}(\lambda) \equiv-\frac{\partial L}{\partial(d t / d \lambda)}=\frac{\kappa c^{2}(d t / d \lambda)}{\sqrt{(d \mathbf{x} / c d \lambda)^{2}-(d t / d \lambda)^{2}}}
$$

can be considered, with respect to the evolution of the parameter $\lambda$, as the energy, which is conserved invariantly like as the energy of the bradyon. When expressed in the three-vector $\mathbf{u}$, $-\Pi^{0}(\lambda)$ becomes

$$
-\Pi^{0}(\lambda)=\frac{\kappa c^{2}}{\sqrt{u^{2} / c^{2}-1}} \equiv E(t),
$$

which is also formally the same result as in the previous section. However, actually the replacement of the functions of $\lambda, \Pi(\lambda)$ and $-\Pi^{0}(\lambda)$ with the functions of $t, \mathbf{p}(t)$, and $E(t)$ is non-trivial matter because $\lambda$ may not be a function of $t$ in the mathematical rigor although $t$ is the function of $\lambda$ generally by having in mind the infinite velocity tachyon (Fig. 10). Hence the replacement of Eqs. (86) and (88) should be understood as $\mathbf{p}(t) \equiv \mathbf{p}(t(\lambda))$ and $E(t) \equiv E(t(\lambda))$. Moreover, in order to prove that they are also the conserved quantities, i.e., constant, with respect to the physical time parameter $t$ " without any ambiguity", let us consider the change of $\mathbf{p}(t(\lambda))$ and $E(t(\lambda))$ in a small interval of $\lambda$ along the world-line

$$
\begin{aligned}
d \mathbf{p}(t(\lambda)) & =\mathbf{p}\left(t_{2}\left(\lambda_{2}\right)\right)-\mathbf{p}\left(t_{1}\left(\lambda_{1}\right)\right) \\
d E(t(\lambda)) & =E\left(t_{2}\left(\lambda_{2}\right)\right)-E\left(t_{1}\left(\lambda_{1}\right)\right)
\end{aligned}
$$

where $\lambda_{2}=\lambda_{1}+d \lambda$. Then, by using the equations of motion (82) and (83) we can easily see that

$$
\begin{aligned}
d \mathbf{p}(t(\lambda)) & =\left(\frac{d \mathbf{p}}{d \lambda}\right) d \lambda=0 \\
d E(t(\lambda)) & =\left(\frac{d E}{d \lambda}\right) d \lambda=0
\end{aligned}
$$

for any $d \lambda$ and hence for any $d t$ implying that $\mathbf{p}$ and $E$ are really the conserved ones with respect to the time $t$ along the world-line for the transient tachyon also as well as the other tachyons. 
Therefore, in this section we have shown that the least action principle can be also applied to the tachyon, and moreover by introducing this principle we have reproduced the essential results for the tachyon of the previous section, i.e., the energy and momentum formula and the mass transformation formula only by considering the single object case and without consideration of the many objects collision processes, which has been essential to derive the results uniquely. This corresponds to a merit of the least action approach. However, for the determination of the zero-point energy of the point object in the previous section, it seems that the elementary approach of that section is more clear and simple, and so this will not be reconsidered in this section. Finally, we note that the results of the tachyon as well as the bradyon have been obtained independently on the existence of the luxon.

\section{THEORY OF THE LUXON}

For the description of the point-like object moving with the light velocity, i.e., luxon, the analysis of the previous two sections can not be directly applied to it, as a limit case of $\lim u \rightarrow c$ without any ambiguity. This can be easily seen by considering, for example, the formula (70) and (74) of the momentum and energy of the bradyon for the case of $\lim u \rightarrow c$ in all uniformly moving systems. In this limit, both $\mathbf{p}$ and $E$ are ill-defined:

a) for non-zero proper-mass luxon, only the infinite value of $\mathbf{p}$ and $E$ are allowed in any systems. This luxon is not believed to be detected in our essentially finite-mass bradyonic detectors, and hence is unphysical objects, and

b) for zero proper-mass luxon, both $\mathbf{p}$ and $E$ has the form of $0 / 0$ and hence they are also ill-defined unless we know the explicit pattern of $m_{0} \rightarrow 0$ as $u \rightarrow c$ [24].

Furthermore, since both $\mathbf{p}$ and $E$ are ill-defined, their any interrelations are also ill-defined such that the usual formula of the luxon

$$
E=p c
$$

can not be justified in "a purely mechanical sense" although it is satisfied for all examples of luxon as far as we know [25]. These facts are also true for the limit $u \rightarrow c$ from the tachyon formulas. Hence, both the bradyon and tachyon have ill-defined limits of $u \rightarrow c$. Actually this fact is not surprising because all derivations of the previous sections II and III can not be justified for the case of the luxon with mathematical rigor. Hence, for the consistent description 
of the luxon, an independent theory is needed.

\section{Least action approach to the luxon dynamics}

With the help of the least action principle, we can naturally and easily construct the theory of luxon without any ambiguity. To this end, we first note that the world-parameter $w$ is not appropriate for the description of the world-trajectory of the luxon because

i) $\Delta w=\Delta t \sqrt{1-u^{2} / c^{2}}=\Delta t \cdot 0=0$ for any time interval $\Delta t$ with $|\Delta t|<\infty$ and

ii) $\Delta w=\Delta t \cdot 0$ has ambiguous value for $|\Delta t|=\infty$.

Here $\Delta w \equiv w_{B}-w_{A}, \Delta t \equiv t_{B}-t_{A}$ are differences of their values for any two events $A$ (leaving of luxon) and $B$ (arriving of luxon) along the world-line. Furthermore, due to this fact we note secondly that from the previous formula (49) the world-velocity $\mathcal{U}^{\mu}$ of the luxon has the indefinite values

$$
\mathcal{U}^{\mu} \equiv \frac{d x^{\mu}}{d w}=\left(\frac{c}{0}, \frac{c \hat{k}}{0}\right)
$$

where $\hat{k}$ is the unit propagation vector and hence $\mathcal{U}^{\mu}$ is not appropriate for the description of the dynamics of the luxon having the well-defined definite velocity by definition.

In order to avoid this problem we introduce a real-valued parameter $\eta$ describing the trajectory of the luxon $x^{\mu}=x^{\mu}(\eta)$ by

$$
\begin{aligned}
\frac{d x^{\mu}}{d \eta} \frac{d x_{\mu}}{d \eta} & =\frac{d x^{\mu}}{d t} \frac{d x_{\mu}}{d t}\left(\frac{d t}{d \eta}\right)^{2} \\
& =\left(c^{2}-u^{2}\right)\left(\frac{d t}{d \eta}\right)^{2} \\
& =0
\end{aligned}
$$

with the condition

$$
\left(\frac{d t}{d \eta}\right)^{2}<\infty
$$

such that Eq. (93) characterizes the case of the luxon. Then, in order that these defining relations are Lorentz-covariantly valid, some restrictions on $\eta$ are needed. By defining the Lorentz transformation of $\eta$ as $\eta^{\prime}=\eta^{\prime}(\eta)$, i.e., the transformation law of $\eta$ under the Lorentz 
transformation can be expressed as is independent of the space and time coordinates of the objects, the relation (93) is covariantly satisfied as follows

$$
\frac{d x^{\mu \prime}}{d \eta^{\prime}} \frac{d x_{\mu}{ }^{\prime}}{d \eta^{\prime}}=\frac{d x^{\mu}}{d \eta} \frac{d x_{\mu}}{d \eta}\left(\frac{d \eta}{d \eta^{\prime}}\right)^{2}=0
$$

only if

$$
\left(\frac{d \eta}{d \eta^{\prime}}\right)^{2}<\infty
$$

,i.e., the Lorentz transformation $\eta^{\prime}=\eta^{\prime}(\eta)$ is a non-singular transformation. Furthermore, the condition (94) is also covariantly satisfied as follows

$$
\begin{aligned}
\left(\frac{d t^{\prime}}{d \eta^{\prime}}\right)^{2} & =\left[\gamma\left(\frac{d t}{d \eta^{\prime}}-\frac{\mathbf{v}}{c^{2}} \cdot \frac{d \mathbf{x}}{d \eta^{\prime}}\right)\right]^{2} \\
& =\gamma^{2}\left(1-\frac{\mathbf{v}}{c^{2}} \cdot \mathbf{c}\right)^{2}\left(\frac{d t}{d \eta}\right)^{2}\left(\frac{d \eta}{d \eta^{\prime}}\right)^{2}<\infty
\end{aligned}
$$

only if Eq. (96) is satisfied. Hence, only if we consider non-singular transformation $\eta^{\prime}=$ $\eta^{\prime}(\eta)$ under the Lorentz transformation, the defining relations (93) and (94) can be satisfied covariantly. Now, by defining the null-velocity vector of the luxon $l^{\mu}$ as

$$
l^{\mu} \equiv \frac{d x^{\mu}}{d \eta}
$$

the possible candidate for the invariant combinations from this velocity vector are $l^{\mu} l_{\mu},\left(l^{\mu} l_{\mu}\right)^{2}$, ..., etc.. Although it seems that the property of

$$
l^{\mu} l_{\mu}=0
$$

make these combinations inappropriate as the building blocks of the Lorentz-invariant action at first, this is not the case because this equation is a dynamical equation in contrast to the nondynamical mathematical identity (59) of the bradyon and the tachyon: Eq.(99) does express that the velocity of the luxon is always the light velocity which being the only mechanical property of the luxon [26]. Hence the action of the luxon, if it exists, should provide the equation of motion (93) or (99).

To realize this scenario we introduce a variable $\xi(\eta)$ into the action of the luxon by

$$
\mathcal{J}\left[\eta_{1}, \eta_{2}\right]=-\frac{1}{2} \int_{\eta_{1}}^{\eta_{2}} l^{\mu} l_{\mu} \xi d \eta
$$


analogous to $K$ in Eq.(64), but now $\xi$ is not the pseudo-scalar or scalar in general rather it's transformation under the Lorentz transformation is the same as " $d \eta$ ", i.e.,

$$
\xi(\eta) \rightarrow \xi^{\prime}\left(\eta^{\prime}\right)=\left(\frac{d \eta^{\prime}}{d \eta}\right) \xi(\eta) \quad \text { when } \quad d \eta \rightarrow d \eta^{\prime}=\left(\frac{d \eta^{\prime}}{d \eta}\right) d \eta
$$

in order that the action (100) is Lorentz invariant. Here the factor $1 / 2$ in front of the action is introduced for convenience. Note that although by varying $\mathcal{J}$ with respect to $\xi$ we can recover the equation of motion of luxon (93) or (99), the variable $\xi$ should not be considered as an auxiliary field as in the case of usual 'einbein' formulation [27]. This means that the equation of motion with respect to variation of $\xi$ should not be implemented back into the action (100) as in the case of auxiliary field: if it is implemented to (100), the action becomes vanishing, which does not show any dynamics of the luxon. So we emphasize that the new variable $\xi$ is not an auxiliary one although there is no derivatives of $\xi$ with respect to $\eta$ in our formulation [28]. Moreover, we note that the Lorentz transformation of $\xi$, Eq.(101) implies the invariance of the action (100) under the reparametrization

$$
\eta \rightarrow \tilde{\eta}(\eta)
$$

if the variable $\xi$ does not distinguish the variation of $\eta$ under the Lorentz transformation and that of under the reparametrization, i.e.,

$$
\xi(\eta) \rightarrow \tilde{\xi}(\tilde{\eta})=\left(\frac{d \tilde{\eta}}{d \eta}\right) \xi(\eta)
$$

Note that this assumption is plausible since $\xi$ is the function of only $\eta$. Hence, the Lorentz invariance implies the reparametrization invariance in this case.

Now let's consider their all equations of motion. By defining the Lagrangian $L(\eta)$ of the luxon with the parameter $\eta$ as

$$
\mathcal{J}\left[\eta_{1}, \eta_{2}\right]=\int_{\eta_{1}}^{\eta_{2}} L(\eta) d \eta
$$

with

$$
L(\eta)=-\frac{1}{2} \xi(\eta) l^{\mu} l_{\mu}=-\frac{1}{2} \xi(\eta)\left[\left(\frac{c d t}{d \eta}\right)^{2}-\left(\frac{d \mathbf{x}}{d \eta}\right)^{2}\right]
$$

the equations of motion become

$$
\begin{aligned}
& 0=\frac{\partial L}{\partial t}-\frac{d}{d \eta}\left[\frac{\partial L}{\partial(d t / d \eta)}\right]=-\frac{d}{d \eta}\left[\frac{\partial L}{\partial(d t / d \eta)}\right] \\
& 0=\frac{\partial L}{\partial \mathbf{x}}-\frac{d}{d \eta}\left[\frac{\partial L}{\partial(d \mathbf{x} / d \eta)}\right]=-\frac{d}{d \eta}\left[\frac{\partial L}{\partial(d \mathbf{x} / d \eta)}\right]
\end{aligned}
$$


from the least action condition

$$
\begin{aligned}
0 & =\delta_{[\eta]} \mathcal{J}\left[\eta_{1}, \eta_{2}\right]=\int_{\eta_{1}}^{\eta_{2}} \delta_{[\eta]} L(\eta) d \eta \\
& =\int_{\eta_{1}}^{\eta_{2}}\left\{\left[\frac{\partial L}{\partial t}-\frac{d}{d \eta}\left[\frac{\partial L}{\partial(d t / d \eta)}\right]\right] \delta_{[\eta]} t+\left[\frac{\partial L}{\partial \mathbf{x}}-\frac{d}{d \eta}\left[\frac{\partial L}{\partial(d \mathbf{x} / d \eta)}\right]\right] \cdot \delta_{[\eta]} \mathbf{x}\right\} d \eta
\end{aligned}
$$

with the auxiliary conditions

$$
\delta_{[\eta]} x^{\mu}\left(\eta_{1}\right)=\delta_{[\eta]} x^{\mu}\left(\eta_{2}\right)=0 .
$$

In this case the quantity

$$
\boldsymbol{\Pi}(\eta) \equiv \frac{\partial L}{\partial(d \mathbf{x} / d \eta)}=\xi \frac{d \mathbf{x}}{d \eta}
$$

can be considered as the canonical momentum, which is conserved with respect to the evolution of parameter $\eta$ in uniformly moving systems from the same reason as in the case of the tachyon. When expressed in the three-vector $\mathbf{u}=c \hat{k}$ with unit propagation vector $\hat{k}, \boldsymbol{\Pi}(\eta)$ becomes

$$
\Pi(\eta)=\left(\frac{d t}{d \eta}\right) \xi c \hat{k}
$$

which is invariant under the reparametrization (102) and (103).

On the other hand, the quantity

$$
-\Pi_{0}(\eta) \equiv-\frac{\partial L}{\partial(d t / d \eta)}=\left(\frac{d t}{d \eta}\right) \xi c^{2}
$$

can also be considered as the conserved energy with respects to the evolution of parameter $\eta$ invariantly, and also is invariant under the reparametrization (102) and (103). Furthermore, since $\eta$ can always be considered as a function of $t$ due to the condition of (94), it is trivial to represent the functions of $\eta, \Pi(\eta)$ and $\Pi_{0}(\eta)$ as the functions of $t$ as

$$
\Pi(\eta) \equiv \mathbf{p}(t), \quad-\Pi_{0}(\eta) \equiv E(t)
$$

such that

$$
\frac{d \mathbf{p}(t)}{d t}=0, \quad \frac{d E(t)}{d t}=0
$$

are satisfied, i.e., $\mathbf{p}(t)$ and $E(t)$ are conserved quantities with respect to the evolution of the time $t$. Note that we have now expressed the dynamical quantities of the luxon without any ambiguity by the function

$$
(d t / d \eta) \xi
$$


which is the reparametrization invariant, and by the propagation vector $\hat{k}$. Hence the essential role of $\xi$ is that it acts as a new dynamical parameter or variable when combined with the quantity $(d t / d \eta)$ for the unambiguous description of the system of the luxon.

Furthermore, it is easy to see that the momentum and energy formulas (110)-(112) really show the usual energy-momentum relation for the luxon

$$
E^{2}-p^{2} c^{2}=0,
$$

and the transformation under the Lorentz transformation

$$
\begin{aligned}
\mathbf{p}^{\prime} & =\xi^{\prime} \frac{d \mathbf{x}^{\prime}}{d \eta^{\prime}}=\xi \frac{d \mathbf{x}^{\prime}}{d \eta} \\
& =\left[1+(\gamma-1) \frac{\mathbf{v}}{v^{2}} \mathbf{v} \cdot\right] \xi \frac{d \mathbf{x}}{d \eta}-\gamma \frac{\mathbf{v}}{c^{2}} c^{2} \xi \frac{d t}{d \eta} \\
& =\left[1+(\gamma-1) \frac{\mathbf{v}}{v^{2}} \mathbf{v} \cdot\right] \mathbf{p}-\gamma \frac{\mathbf{v}}{c^{2}} E, \\
E^{\prime} & =c^{2} \xi^{\prime} \frac{d t^{\prime}}{d \eta^{\prime}}=c^{2} \xi \frac{d t^{\prime}}{d \eta} \\
& =\gamma\left(c^{2} \xi \frac{d t}{d \eta}-\xi \frac{d \mathbf{x}}{d \eta} \cdot \mathbf{v}\right) \\
& =\gamma(E-\mathbf{p} \cdot \mathbf{v})
\end{aligned}
$$

according to the Lorentz transformation (101) of $\eta$ and $\xi$ without any ambiguity, which has been impossible with mathematical rigor due to the ill-defined nature of the energy and momentum of the bradyon and tachyon for the luxon limit.

Now, with the help of this energy and momentum transformation, we can easily prove by considering the collision process of the arbitrary number, and kind of the luxons should satisfy the same constraints as in the case of the tachyon and bradyon of Sec. II if we maintain the definition of the energy and momentum as the conserved quantities for all the systems of coordinates and all the possible process of the collision. Especially, for the case of the photon it's zero-point energy should be zero due to the fact that it is clearly " the strictly neutral particles ". Furthermore, due to the transformation (117) we can also define the four-momentum

$$
p^{\mu}=(E, c \mathbf{p})=\left(c^{2} \xi \frac{d t}{d \eta}, c \xi \frac{d \mathbf{x}}{d \eta}\right)=c \xi \frac{d x^{\mu}}{d \eta},
$$


which is the light-like, i.e.,

$$
p^{\mu} p_{\mu}=p^{\mu \prime} p_{\mu}^{\prime}=0
$$

due to Eq. (116), and hence conservation law (114) can be expressed as a covariant form

$$
\frac{d p^{\mu}}{d t}=0
$$

It is interesting to note that the luxon exists only as a quantum object in our universe as far as we know. In this case, with the help of the Einstein relation for the photon or neutrino

$$
E=\hbar c k, \quad \mathbf{p}=\hbar k \hat{k}
$$

the function (115) is found to be

$$
(d t / d \eta) \xi=\hbar k
$$

\section{SUMMARY AND CONCLUDING REMARKS}

In this paper we have presented the classical foundation of the relativistic dynamics including

the tachyon. An anomalous sign-factor in the transformation of $\sqrt{1-u^{2} / c^{2}}$ under the Lorentz transformation, which is unimportant for the bradyon and has been always missed in the usual formulation of the tachyon, has now a crucial role in the dynamics of the tachyon. Due to this novel sign-factor, it is found from the consideration of the collision process that the proper-mass of the tachyon is not an absolute quantity in contrast to that of the bradyon, but transforms as $\kappa^{\prime}=\operatorname{sign}\left\{1-\mathbf{u} \cdot \mathbf{v} / c^{2}\right\} \kappa$. Furthermore, we have shown that the tachyon's mass has a definite sign for a given speed in a uniformly moving system, but has the sign changed depending on the tachyon's velocity and the relative motion of the systems $S$ and $S^{\prime}$.

On the other hand, by defining the momentum and energy as the conserved quantities of the form Eq. (22) and (24) in all uniformly moving systems, we have shown that the zero-point energy $\epsilon_{a}$ for any kind of the objects of the both tachyon and bradyon, which has been known as the undetermined constant, should satisfy some constraints ,i.e., $\epsilon_{a}=0$ for the strictly neutral particles and $\epsilon_{a}$ particle $=-\epsilon_{a}$ antiparticle in other cases however remainly undetermined for the latter case for consistency. Furthermore, especially for the case of $\epsilon_{a} \equiv 0$ for all $a$ as in the usual conventions, it is found that the energy and momentum for the tachyon as well as the bradyon satisfy the usual four-vector transformation such that the energy for the tachyon does not have the invariant sign of the energy, but the sign can be changed depending on the object's 
velocity and the relative motion of the two systems of coordinates $S$ and $S^{\prime}$ in contrast to that of the bradyon. However, we have noted that this transformation formulas can not be obtained from the usual formulation of the tachyon in contrast to the usual belief such that the original motivation of the BDSF's reinterpretation principle, if it applies to our Nature, can not be found in the usual formulation but only in our new formulation of the tachyon.

As an alternative approach we have also presented the least action approach to the tachyon dynamics. Although it is not clear whether this approach can be also applied to the tachyon since the situation of $\Delta t \equiv 0$ for the time interval along the world-line can not be avoided for any tachyon such that there are some ambiguities in deriving the equations of motion. However, we have shown that this can be really also applied to the tachyon with the help of the reparametrization invariance of the action, and the essential results for the tachyon of the elementary approach of Sec. II can be also rederived in this approach.

Furthermore, we have shown that an unambiguous description of the luxon dynamics is possible with the help of the least action approach, which has not been clear in the approach of Sec. II. In this approach, to this end a new dynamical variable $\xi$ with the parameter $\eta$ of the luxon's world-line should have been introduced to the action and with the help of these new variables, the usual energy-momentum relation for the luxon (116) is proved without any ambiguity. Furthermore, it is shown that in this approach the energy and momentum of the luxon also satisfy the usual transformation like as the tachyon and bradyon, which has not been proved in the usual classical mechanical formulation. Moreover, with the help of this energy and momentum transformation under the Lorentz transformation, the zero-point energy for any kind of the luxons should also satisfy the same constraints as in the case of the tachyon and bradyon of Sec. II, if we maintain the definition of the energy and momentum as the conserved quantities for all the systems of coordinates and all the possible process of the collision like as the case of the tachyon and bradyon. Especially, for the case of the photon it's zero-point energy should be zero due to the fact that it is clearly " the strictly neutral particles ". Moreover, we have noted that the luxon exists only as a quantum object as far as we know, and hence in that case the newly introduced variables $\xi$ combined with $\eta$ are related with the variables of the wavicle.

Irrespective of these results, there are several problems for the tachyon, which should be clarified, as a) the problem of the causality violation $[2,3,4]$, b) the problem of the Cerenkov effect in the vacuum $[29,30]$, and c) the problem of the quantization $[2,9]$.

For the causality violation by noting that this effect is essentially due to the interaction between the tachyon and bradyon $[2,3,4]$, we are questioning whether some conditions on the interaction modes, which obstruct the measurement of the effect, can be obtained from the detailed analysis of their interaction. Or the usual concept of the space and time, which are 
defined by the measuring rods and clocks at rest relatively to that system " without references " to the motions, and the properties of the measured object may not be applied to the case of the tachyon, and be needed modification in order to recover the causality in this case also. On the other hand, in our opinion it is questionable whether the principle of causality can be considered as the fundamental principle of our Nature like as the principles of the theory of relativity, and hence we might think that the communication via the bradyon or luxon is a special case of the preservation of causality but not true in general with the tachyon.

For the Čerenkov effect in the vacuum, there has been no complete agreement for the effect in relation mainly with the non-covariance of this effect, i.e., " the transient tachyon problem " [8,30], which states that the transient charged tachyon, i.e., zero energy (considered as the lowest energy) tachyon does not radiate, but some radiation should be observed in other systems due to the non-invariance of the property of the transient tachyon such that the principle of relativity is violated if there exist Čerenkov effect in the vacuum. But, here we note that this may be not the case essentially because in the case of the tachyon the zero energy is not the lowest energy state of it. Rather there are the lower states, i.e., the negative energy states such that the zero energy tachyon may also radiate if there is the Cerenkov radiation for the positive energy tachyon. The reason for the inclusion of the negative energy states into the energy range of the tachyon is that firstly, the tachyons having different sign of the energy and hence of the mass are connected by the Lorentz transformation, and secondly there is no energy gap in the energy spectrum in contrast to the case of the bradyon (Fig. 11). However, it is not clear whether this modified view can provide the consistent covariant theory of the Čerenkov effect. Furthermore, we note that the effect depends on the microscopic structure of the tachyon like as the self-energy problem such that we can imagine the complete understanding can be obtained only after the quantization. So far our understanding for the Cerenkov effect has been restricted to the usual coupling, i.e., minimal couplings. So it would be interesting to study the Čerenkov effect for the non-minimal coupling for the tachyon. However, we note that the minimal coupling is remainly good coupling for the case of the "charged" luxon as in the case of the bradyon. In this case, by considering the Lorentz invariant electric charge $q$ of the luxon [31], the total action becomes

$$
\begin{aligned}
\mathcal{J}\left[\eta_{1}, \eta_{2}\right] & =\int_{\eta_{1}}^{\eta_{2}}\left[-\frac{1}{2} \xi(\eta) l^{\mu} l_{\mu} d \eta-\frac{q}{c} A_{\mu}\left(x_{p}(\eta)\right) d x_{p}^{\mu}\right] \\
& =\int_{\eta_{1}}^{\eta_{2}}\left[-\frac{1}{2} \xi(\eta) \frac{d x^{\mu}}{d \eta} \frac{d x_{\mu}}{d \eta} d \eta-\frac{q}{c} A_{\mu}\left(x_{p}(\eta)\right) d x_{p}^{\mu}\right]
\end{aligned}
$$

for the particle position $x_{p}^{\mu}(\eta)$, and the equation of motion becomes

$$
\frac{d}{d \eta}\left[\frac{d x_{\nu}}{d \eta} \xi(\eta)\right]=\frac{q}{c} F_{\nu \mu} \frac{d x^{\mu}}{d \eta},
$$


or with the physical time $t$

$$
\frac{d}{d t} P_{\nu}=q F_{\nu \mu} \frac{d x^{\mu}}{d t}
$$

where $d x^{\mu} / d t=c(1, \hat{k})$. Note that this interaction does not induce the Cerenkov radiation in vacuum.

Now, for the quantization, it has been noted that a new method of quantization is needed in the field quantization theory for the tachyon, but no consistent method has been suggested so far $[2,9]$. Here we note that more fundamentally the quantum theory of the tachyon, if it exists, is different from the usual quantum theory of the bradyon by considering the measurement of the momentum and coordinate of a tachyon.

To this end we first note that for the coordinate measurement at a time $t$ the uncertainty of the coordinate $\Delta \mathbf{x}$ for one object is related by the uncertainty of the momentum $\Delta \mathbf{p}$ as

$$
\Delta \mathbf{x}(t) \geq \frac{\hbar}{\Delta \mathbf{p}(t)}
$$

according to the Heisenberg's uncertainty principle. Then, it is easy to see that for the bradyon there is a lower bound for the magnitude of the right-hand side of the relation (126) as $\hbar /\left(2 m_{0} c\right)$ [32] since i) for the one object case, $2 m_{0} c$ is the maximum uncertainty of the momentum for it's validity according to the usual particle and anti-particle theory indirectly due to the existence of the maximum uncertainty for the energy [33], and ii) for the many object case, i.e., when the many object and anti-object pairs are produced from the high momentum transfer $\Delta \mathbf{p}$ in the measurement process, $\Delta \mathbf{x}$ (uncertainty of coordinate for each object) behaves as $\sim \Delta \mathbf{X} / N$ as $\Delta \mathbf{P}$ behaves as $\sim N m_{0} c(\Delta \mathbf{X}$ and $\Delta \mathbf{P}$ represent the uncertainty of the coordinate and momentum for the total system of the many objects respectively). Hence, in the quantum theory it is, in principle, impossible to make an arbitrarily accurate measurement of the coordinate of the bradyon. This property is still satisfied even by the tachyon although the situation is different. In this case there is also a lower bound directly due to the existence of the maximum uncertainty of the momentum for maintaining the number of the objects although there is no maximum uncertainty for the energy in that case (Fig. 11, 12) both for the one object case and many object case similarly to the analysis of the bradyon. Hence, as for the measurement of the coordinate the tachyon and bradyon behave similarly, i.e., it is, in principle, impossible to make an arbitrarily accurate measurement of the coordinate for the case of the both tachyon and bradyon [34].

However, this is not case for the momentum measurement. To see this we note that, following the argument of Landau and Peierls [32], for the momentum measurement during a 
time interval $\Delta t$, the uncertainty of the momentum $\Delta \mathrm{p}$ is related as

$$
\Delta \mathbf{p} \Delta t \geq \frac{\hbar}{\left(\mathbf{u}-\mathbf{u}^{\prime}\right)}
$$

by applying the Heisenberg's uncertainty principle, where $\mathbf{u}$ and $\mathbf{u}^{\prime}$ are the velocities of the tachyon before and after the measurement. Now it is easy to see that, for the bradyon, there is a lower bound for the right-hand side of the relation (127) as $\hbar / c$, due to the velocity limit of the bradyon $c$, such that in the relativistic quantum theory it is, in principle, impossible to make an arbitrarily accurate and rapid measurement of the momentum. On the other hand, this is not true for the case of the tachyon since in this case there is no upper velocity limit such that any arbitrarily accurate and rapid measurement of the momentum.

Hence in the quantum theory, if it exists, the momentum of the tachyon can act as the dynamical variable in contrast to the case of the relativistic bradyon such that the physical significance of the momentum-space wavefunction $\varphi(\mathbf{p})$ is as important as that of the nonrelativistic bradyon. However, the coordinate of the tachyon can not act as the dynamical variable similar to the case of the relativistic bradyon such that the wavefunction $\psi(\mathbf{x}, t)$ is not as important as that of the non-relativistic case. In this sense the quantum theory of the tachyon resembles that of the non-relativistic quantum theory of the bradyon in one aspect, i.e., momentum measurement, but resembles that of the relativistic quantum theory of the bradyon in another aspect, i.e., coordinate measurement. In other words, the quantum theory of the tachyon, if it exists, would be a mixture of the relativistic and non-relativistic quantum theory of the bradyon. Furthermore, we are questioning whether, due to these peculiar properties, the divergence problem of the usual quantum field theory of the bradyon does not appear in the case of the tachyon since the problem is not independent on the limit of the accuracy for the measurement of the coordinate and the momentum.

Finally, we hope that through further investigation the consistent dynamics of the tachyon including the interaction will be established.

\section{ACKNOWLEDGMENTS}

The present work was supported by the Basic Science Research Institute program, Ministry of Education, Project No. BSRI-95-2414. 


\section{REFERENCES}

1. It should be understood hereafter that all our analysis are made in "vacuum " though not specified explicitly.

2. S. Tanaka, Prog. Theor. Phys. 24 (1960), 171; O. M. P. Bilaniuk, V. K. Deshpande, and E. C. G. Sudarshan, Am. J. Phys. 30 (1962), 718; G. Feinberg, Phys. Rev. D 159 (1967), 1089 .

3. A. Einstein, Ann. Phys. 17 (1905), 891.

4. A. Einstein, Jahrb. Radioaki. 4 (1907), 411; 5(E) (1907), 98. The English version can be obtained from "The Collected Papers of Albert Einstein " ( A. B. Beck and P. Havas, Eds.), Vol. 2, p. 252, Princeton University Press, Princeton, New Jersey, 1989.

5. R. G. Cawley, Ann. Phys. 54 (1969), 122; Phys. Rev. D 2 (1970), 276; E. Recami, Accad. Naz. Lincei Rendic. Sc. 49 (1970), 77.

6. A. Einstein, "Relativity", 15th ed., p. 35, Crown Publishers, Inc., New York, 1952; A. I. Miller, "Albert Einstein's Special Theory of Relativity ", p. 331, Addison- Wesley Publishing Company, Inc., Sydney, 1981.

7. This is because the ordinary detectors will get some imaginary energy or momentum by detecting the tachyon since the process of the measurement is essentially due to the exchange of the energy or momentum in a detectable time interval.

8. O. M. Bilaniuk and E. C. G. Sudarshan, Nature, 223 (1969), 386; O. M. Bilaniuk et. al, Phys. Today 22 (No.12) (1969), 47; R. G. Newton, Science 167 (1970), 1569 and references therein.

9. M. E. Arons and E. C. G. Sudarshan, Phys. Rev. 173 (1968), 1622; J. Dhar and E. C. G. Sudarshan, ibid. 174 (1968), 1808; G. Ecker, Ann. Phys. 58 (1970), 303; B. Schroer, Phys. Rev. D 3 (1971), 1764; K. Kamoi and S. Kamefuchi, Prog. Theor. Phys. 45 (1971), 1646; C. Jue, Phys. Rev. D 8 (1973), 1757.

10. D. Korff and Z. Fried, Nuov. Cim. 52 (1967), 173; Y. P. Terletskii, "Paradoxes in The Theory of Relativity", p. 88, Plenum Press, New York, 1968.

11. O. M. Bilaniuk and E. C. G. Sudarshan, Phys. Today 22 (No.5) (1969), 43. 
12. The reverse of this reasoning is also true, i.e., if Eq. (3) (energy) is defined to the totally conserved quantity, mass $m(\mathbf{u})$ is found as Eq. (2), and Eq. (1) (momentum) is proved to be totally conserved. See, for example, R. Penrose and W. Rindler, Am. J. Phys. 33 (1965), 55; J. Ehlers, W. Rindler, and R. Penrose, ibid. 33 (1965), 995.

13. P. G. Bergmann, "An Introduction to the Theory of Relativity ", p. 87, Prentice-Hall International, Inc., London, 1942; C. Møller, "The Theory of Relativity ", p. 67, Oxford University Press, Oxford, London, 1952); M. Born, "Einstein's Theory of Relativity", p. 267 , Dover Publications, Inc., New York, 1962.

14. G. N. Lewis and R. C. Tolman, Phil. Mag. 18 (1909), 510; R. C. Tolman, ibid. 23 (1912), 375; "Relativity, Thermodynamics and Cosmology ", p. 42, Oxford University Press, Oxford, England, 1934; A. Einstein, Amer. Math. Soc. Bull. April (1935), 223; W. H. McCrea, "Relativity Physics", p. 19, Methuen and Company, Ltd., London, 1935; D. Bohm, " The Special Theory of Relativity", p. 81, W. A. Benjamin, Inc., New York, 1965; R. K. Pathria, "The Theory of Relativity ", 2nd ed., p. 77, Pergamon Press Ltd., Headington Hill Hall, Oxford, 1974; D. S. Mann and P. K. Mukherjee, “ Relativity, Mechanics and Statistical Physics ", p. 50, John Wiley and Sons, Inc., New York. The full review of the various other formulations as well as afore-mentioned the two can be obtained from H. Arzeliès, "Relativistic Point Dynamics ", p. 19, Pergamon Press, Oxford, 1971.

15. H. Arzeliès [14].

16. L. Parker, Phys. Rev. 188 (1969), 2287; E. Recami and R. Mignani, Lett. Nuov. Cim. 4 (1972), 144; A. F. Antippa, Nuov. Cim. A 10 (1972), 389; R. Goldoni, ibid. 13 (1973), 501; 527; H. C. Corben, ibid. 29 (1975), 415.

17. V. B. Berestetskii, E. M. Lifshitz, and L. P. Pitaerskií, "Relativistic Quantum Theory ", Pergamon Press, New York, 1971.

18. Although several authors in Ref.[10] pointed out the incompleteness of the usual formulation of BDSF with regard to the problem of the conservation law of momentum and energy, they did not provide the complete solution to this problem in contrast to their claims.

19. L. D. Landau and E. M. Lifshitz, "The Classical Theory of Fields ", 4th English ed., Pergamon Press, Oxford, 1975; S. Weinberg, "Gravitation and Cosmology ", John Wiley \& Sons, New York, 1972; F. Rohrlich, "Classical Charged Particles ", Addison-Wesley Publishing Company, Inc., Massachusetts, 1965. 
20. Although the pseudo-tensor $T^{\mu \nu \ldots \omega}$ is defined usually as $T^{\mu \nu \ldots \omega}=\alpha \Lambda_{\mu^{\prime}}^{\mu} \Lambda_{\nu^{\prime}}^{\nu} \ldots \Lambda^{\omega}{ }_{\omega^{\prime}} T^{\mu^{\prime} \nu^{\prime} \ldots \omega^{\prime}}$, $\alpha=\operatorname{det}\left(\Lambda^{\mu}{ }_{\nu}\right)= \pm 1$ with the orthogonal transformation matrix $\Lambda^{\mu}{ }_{\nu}$, we introduce here more general definition of it having the arbitrary sign-factor $\alpha$ which is not necessarily the $\operatorname{det}\left(\Lambda^{\mu}{ }_{\nu}\right)$. In our case $\alpha$ is the anomalous sign-factor " $\operatorname{sign}\left\{1-\mathbf{u} \cdot \mathbf{v} / c^{2}\right\} "$.

21. E. C. G. Stüeckelberg, Helv. Phys. Acta. 14 (1941), 588; ibid. 15 (1942), 23( We thank Jyung-Youn Choi for English translation from French); R. P. Feynman, Phys. Rev. 74 (1948), 939; ibid. 76 (1949), 749.

22. For the free object, the imaginary-valued action may be allowed with slight modification of the action principle without changing the physical contents. However, only the realvalued action is meaningful if we consider the physically meaningful interactions between this system and the system of the bradyon such that the imaginary-valued momentum and energy does not appear in the theory if the action of the bradyon is defined to be real-valued as usual.

23. Although it seems that the derivation of Eq. (76) has some ambiguities since we must use the relation $(d \lambda / d t) d t=d \lambda$, which is ambiguous for $d t=0$ case. But this is actually not the case because the last formula exactly reproduce the first one even in this case.

24. Of course, the explicit pattern of $m_{0} \rightarrow 0$ as $u \rightarrow c$ can not be given according to the results of the previous two sections because $m_{0}$ is defined to be Lorentz constant without the functional dependence of any other variable, like as it's velocity.

25. Of course, it is possible to prove that this formula by using the Maxwell equation is satisfied for the chunks of the electromagnetic radiation. See, for example, C. Møller [13] and Ref. [19].

26. Here we are not considering the internal spin quantity for the luxon.

27. M. B. Green, J. H. Schwartz and E. Witten, "Superstring Theory " Vol. 1, Cambridge University Press, Cambridge, 1987; A. M. Polyakov, "Gauge Fields and Strings ", Harwood Academic Publishers, England, 1987.

28. In general, it is expected that the action produces trivial or non-dynamical information about the interesting system if we implement the equations of motion with respect to variation of some dynamical variables back into the action. This is essentially due to the fact that the equations of motion are nothing but the conditions for the stable point of the action, which is non-dynamical. 
29. A. Sommerfeld, Proc. Roy. Acad. Amsterdam, 7 (1904), 346; P. A. Čerenkov, Phys. Rev. 52 (1937), 378 and references therein; I. M. Frank and I. E. Tamm, C. R. Ac. Sci. U.S.S.R. 14 (1937), 109; E. C. G. Sudarshan [2].

30. There have been several controversies about the Črenkov effect in the vacuum. E. C. G. Sudarshan, Ark. Phys. 39 (1970), 40; T. Alväger and M. N. Kreisler, Phys. Rev. 171 (1968), 1357; F. Jones, Phys. Rev. D 6 (1972), 2727; H. K. Wimmel, Lett. Nuov. Cim. 1 (1971), 645; Nature Phys. Sci. 236 (1972), 79; R. Mignani and E. Recami, Lett. Nuov. Cim. 7 (1973), 388.

31. Although, as far as we know, there is no electrically charged luxon, we are considering this object since there is no reason to reject this possibility.

32. L. D. Landau and R. Peierls, Z. Phys. 69 (1931), 56; L. D. Landau and E. M. Lifshitz, " Quantum Mechanics ”, §44, Addison-Wesley, Reding, Massachusetts, 1977.

33. By using the energy-momentum relation for the bradyon $E=\sqrt{p^{2} c^{2}+m_{0}^{2} c^{4}}$, the inequality $1 / \Delta p \geq c / \Delta E>1 /\left(2 m_{0} c\right)$ is resulted due to the existence of the maximum uncertainty of the energy for the validity of one object case.

34. This property has been already known in different context, A. Peres, Lett. Nuov. Cim. 1 (1969), 837. 


\section{FIGURES CAPTIONS}

Fig. 1 : For the case of the bradyon, all the directions of the velocity of the moving object relative to the rest object in $S$ are equivalent due to the isotropy of the space. So, if there is the mass relation between the objects moving relative to the rest one for the same kind of objects, the transformation function should not depend on the direction of the velocity of the moving object.

Fig. 2: For the case of the tachyon, all the directions of the velocity of the general tachyons relative to the tachyon with $u_{a}^{2}=2 c^{2}$ in $S$ are not equivalent due to the asymmetry of the situation. So, if there is any relation between the masses of the general tachyons moving relative to the tachyons with $u_{a}^{2}=2 c^{2}$ for the same kinds, the function can be depend on the velocity of the tachyon relative to the tachyon with $u_{a}^{2}=2 c^{2}$ in general.

Fig. 3 : If the mass of the tachyon depends on the direction of the velocity for a given speed, it should be also changed for the different orientations of the coordinates. But this is not allowed in order that the total energy and momentum of Eqs. (4) and (5) are conserved in any orientation of coordinates because the bradyon's mass is independent on the direction of the velocity.

Fig. 4 : In order that the momentum is also conserved in the system $S^{\prime}, \sum_{a} \epsilon_{a}$ should be conserved, i.e., $\sum_{a_{i}=1}^{M} \epsilon_{a_{i}}=\sum_{a_{f}=1}^{N} \epsilon_{a_{f}}$ according to Eq. (25) or (26), as well as the energy and momentum conservation.

Fig. 5 : For the bradyonic world-line, the world-velocity $u^{\mu}$ is time-like, i.e., $u^{\mu} u_{\mu}=c^{2}>0$, while the world-acceleration $a^{\mu}$ is space-like, i.e., $a^{\mu} a_{\mu}=-a^{2} c^{2}\left\{1-\left(u^{2} / c^{2}\right) \sin ^{2} \phi\right\} /\left\{c^{2}(1-\right.$ $\left.\left.u^{2} / c^{2}\right)^{3}\right\}<0$ for any angle $\phi$ between $\mathbf{a}$ and $\mathbf{u}$.

Fig. 6 : For the tachyonic world-line, the world-velocity $t^{\mu}$ is space-like, i.e., $t^{\mu} t_{\mu}=$ $-c^{2}<0$, while the world-acceleration $a_{t}^{\mu}$ is space-like or time-like or light-like, i.e., the sign of $a_{t}^{\mu} a_{\mu_{t}}=-a^{2} c^{2}\left\{1-\left(u^{2} / c^{2}\right) \sin ^{2} \phi\right\} /\left\{c^{2}\left(1-u^{2} / c^{2}\right)^{3}\right\}$ depends on the angle $\phi$ for a given velocity $\mathbf{u}$.

Fig. 7 : Virtual variation of the bradyonic trajectory in the space-time diagram with the conditions $\delta \mathbf{x}\left(t_{1}\right)=\delta \mathbf{x}\left(t_{2}\right)=0$. The virtual variation $\delta x$ defines the variation with fixed time $t$ such that $\delta(d \mathbf{x} / d t)=d(\delta \mathbf{x}) / d t$. Furthermore, the variations are restricted to the time-like cone.

Fig. 8 : Usual variation when applied to the tachyonic trajectory. However, this method with fixed time is doubtful for the infinite velocity tachyon, where the time interval always 
vanish such that there are some ambiguities in deriving the equations of motion. Furthermore, since any tachyon can be made to have the infinite velocity by choosing an appropriate system of coordinates, this problem can not be avoided for any tachyon.

Fig. 9 : Variation in $(\lambda, x)$ space for the tachyonic trajectory. In this space there are no ambiguities for any tachyon: the ambiguity at $u=\infty$ is transferred to the ambiguity at $u=c$, i.e., the case of the luxon such that there is no problem for the tachyon. The allowed trajectories are restricted to the space-like cone except $\lambda=0$ plane.

Fig. 10: A possible function dependence of $t$ and $\lambda$ for the infinite-velocity tachyon according to the relation (43). In this case the physical time $t$ is (constant) function of $\lambda$, but $\lambda$ is not a function of $t$ in mathematical sense.

Fig. 11 : There is no energy gap in the energy spectrum of the tachyon $((b))$ in contrast to the energy gap $2\left|m_{0}\right| c^{2}$ for the bradyon ((a)).

Fig. 12 : There exists the threshold momentum $\kappa c$ for the creation of the tachyon $((b))$ in contrast to the case of the bradyon ((a)). 
This figure "fig1-1.png" is available in "png" format from: http://arxiv.org/ps/hep-th/9506082v1 
This figure "fig1-2.png" is available in "png" format from: http://arxiv.org/ps/hep-th/9506082v1 
This figure "fig1-3.png" is available in "png" format from: http://arxiv.org/ps/hep-th/9506082v1 
This figure "fig1-4.png" is available in "png" format from: http://arxiv.org/ps/hep-th/9506082v1 
This figure "fig1-5.png" is available in "png" format from: http://arxiv.org/ps/hep-th/9506082v1 
This figure "fig1-6.png" is available in "png" format from: http://arxiv.org/ps/hep-th/9506082v1 
This figure "fig1-7.png" is available in "png" format from: http://arxiv.org/ps/hep-th/9506082v1 
This figure "fig1-8.png" is available in "png" format from: http://arxiv.org/ps/hep-th/9506082v1 
This figure "fig1-9.png" is available in "png" format from: http://arxiv.org/ps/hep-th/9506082v1 
This figure "fig1-10.png" is available in "png" format from: http://arxiv.org/ps/hep-th/9506082v1 
This figure "fig1-11.png" is available in "png" format from: http://arxiv.org/ps/hep-th/9506082v1 
This figure "fig1-12.png" is available in "png" format from: http://arxiv.org/ps/hep-th/9506082v1 
This figure "fig1-13.png" is available in "png" format from: http://arxiv.org/ps/hep-th/9506082v1 
This figure "fig1-14.png" is available in "png" format from: http://arxiv.org/ps/hep-th/9506082v1 